\title{
Neural correlates of motor expertise: Extensive motor training and cortical changes
}

\author{
Claire Calmels*
}

French National Institute of Sport (INSEP), Research Department, Laboratory of Sport, Expertise, and Performance (EA 7370), Paris, France

https://doi.org/10.1016/j.brainres.2019.146323

\section{H I G H L I G H T S}

- Studies targeting cortical changes that occur with motor expertise are reviewed.

- Changes at local and network levels occur induced by lenghty-extensive training.

- Cortical changes in experts are seen in areas related to the execution of the task.

- Coexistence of different patterns between various areas makes it arduous to interpret.

- sMRI and fMRI studies probing changes in large-scale complex networks are recommended.

\section{A R T I C L E I N F O}

\section{Keywords:}

Motor expertise

Cortical changes

Extensive and intensive training

SMRI

fMRI

\begin{abstract}
A B S T R A C T
Over the last two decades, there were many investigations on motor expertise but inconsistencies across findings exist. Thus, to unravel these discrepancies, we conducted a novel literature review, applying stricter inclusion criteria relative to those used in previous reviews. Consequently, this paper reviews the most recent MRI and fMRI literature which investigated structural and functional changes underlying motor expertise, defined as being the result of intensive and extensive motor training reflected by a sheer volume of at least several thousand hours. Changes were documented on a local level and on a network level where interactions between pairs of brain components were mainly considered. This review reveals that changes occur induced by lengthy-extensive motor training and that this training shapes the human brain in areas related to the execution of the task. It also highlights the coexistence of different cortical patterns within and between various brain areas/systems which makes it difficult to interpret. Using a large-scale complex network approach based on graph theory facilitates the identification of organizational patterns in brain network and thus enable interpretation. Other recommendations for future research are: attention to participant recruitment, use of histological/biochemical techniques, and combination of sMRI and task-free/task-related fMRIs.
\end{abstract}

\section{Introduction}

Who has not been fascinated by the grace and the apparent ease of a gymnast performing a double somersault or the fluidity and finger speed of a pianist playing a piece of music? While their performances generally seem so easy and effortless, one can forget that the gymnast or the pianist has dedicated a large amount of time, energy, resources and effort to master these tasks to the point of perfection. These people are recognized as experts in the motor domain. They are able to reproduce superior performances with outstanding levels of consistency, stability, and quality. These superior performances are the result of extended and intensive periods of practice which lead to cognitive, motor, physiological, and neural changes. It is widely accepted that becoming an expert in a particular field requires several thousand hours of grueling efforts and practice (Ericsson and Lehmann, 1996; Jancke, 2009; Miall, 2013). Still, a question commonly asked is: which attributes differentiate (motor) expert performers from non-experts?

The advent of neuroimaging techniques has provided insights into the neural mechanisms underlying motor expertise since these enable study of how the brain changes as a result of experience (i.e. learning and training). Changes can occur at structural and functional levels. Structural changes reflect changes in the neuroanatomical architecture (e.g., changes in cortical thickness, in gray and white matter tissues, in cortical networks). Conversely, functional changes describe changes in

\footnotetext{
*Address: Unité Recherche, Laboratoire SEP (EA 7370), Institut National du Sport, de l'Expertise et de la Performance, 11 Avenue du Tremblay, 75012 Paris, France.

E-mail address: claire.calmels@insep.fr.
} 
the recruitment of neuronal tissues (e.g., changes in neuronal activity/ connectivity) when an individual is involved in a specific cognitive, motor, or resting-state activity. In a resting-state activity, subjects are at rest and not performing any explicit task. Contemporary neuroimaging technologies, such as voxel-based morphometry (VBM) or diffusion MRI (e.g., Diffusion Tensor Imaging: DTI and Diffusion Weighed Imaging: DWI), have significantly contributed to the study of structural brain matter architecture in healthy subjects (for further descriptions of these techniques see Box 1). On the other hand, functional cortical changes can be observed via functional MRI (fMRI) which enables visualization of the areas of the brain that are activated during the execution of a specific task or during rest (for further descriptions of the fMRI technique see Box 1).

\section{Box 1}

Descriptions of VMB, Diffusion MRI, and fMRI technologies.

Simply said, VBM is a structural MRI (sMRI) technique that involves a voxel-wise comparison of the local concentration of gray or white matter generally between two groups of subjects or within a group (Mechelli et al., 2005). Two types of analyses are distinguished: non-modulated VBM and modulated VBM. The former analysis reveals differences in the relative concentration or density of gray or white matter (i.e., the proportion of gray or white matter relative to other kinds of tissues within an area) whereas the latter reveals differences in volume (i.e., the absolute amount of gray or white matter in different areas) (Mechelli et al., 2005). Diffusion MRI is also a sMRI technique that enables mapping of the diffusion process of water molecules which can therefore reveal microscopic details about brain tissue architecture (Hagmann et al., 2006). The diffusion criterion employed most frequently is the fractional anisotropy (FA) which appraises the directionality of diffusion within a voxel and the diffusivity trace which assesses the amount of diffusion. FA, which is generally seen as a marker of the quality of white matter fiber tracts or white matter integrity (Alexander et al., 2007; Assaf and Pasternak, 2008), is a complex index since it expresses diverse structural properties of the white matter tissue such as axonal density and diameter, myelination and fibre complexity (Beaulieu, 2002). Finally, computation of the cortical thickness can provide important information about structural brain architecture that is complementary to that collected and calculated via other structural MRI analyses (Hutton et al., 2008). Over the last years, investigation of structural connectivity has rapidly expanded. It refers to the existence of white matter fiber tracts which directly connect different brain areas and allow these areas to communicate. This expansion is due to the development of the DTI and the advent of the graph theory in the field of neurosciences to assess brain networks (Barabasi and Albert, 1999; Bullmore and Sporns, 2009; Watts and Strogatz, 1998). Graph theory provides a mathematical framework to describe, quantify, and model the properties of large system of interconnected elements.

By contrast, fMRI provides an opportunity to investigate noninvasively regional activation or inter-regional connectivity when an individual performs a motor or cognitive task during a scan. Regional activation reflects local changes of activation in particular brain areas: it identifies areas whose activity fluctuates with the task at hand. In contrast, inter-regional connectivity, which has grown in popularity over the last few years, investigates how areas work together as a neural network. Two kinds of connectivity have been distinguished: functional connectivity and effective connectivity (Friston, 1994, 2011). Functional connectivity refers to the degree of association between two areas which can be evaluated by conducting an independent component analysis (ICA) (McKeown et al., 1998) or a seed-based analysis. Effective connectivity refers to the influence one brain area exerts over another one and can be assessed via structural equation modeling (SEM) (Buchel and Friston, 1997; McIntosh and Gonzalez-Lima, 1994), dynamic causal modeling (DCM) (Friston et al., 2003; Stephan et al., 2007), or Granger causal analysis (GCA) (Goebel et al., 2003; Roebroeck et al., 2005). In addition to conventional task-related fMRI, brain activity during resting states has recently been investigated to detect functional changes induced by training and experience (e.g., Di et al., 2012; Luo et al., 2012; Ma et al., 2011; Taubert et al., 2011; Xiong et al., 2009). It has been shown that the topography of functional networks when at rest closely mirrored that of the functional networks at play during the performance of tasks (Lewis et al., 2009; Smith et al., 2009). Likewise, as seen in the investigation of structural brain matter architecture, a network approach has been flourishing over the last years to probe functional network organization. This approach using concepts such as graph, clustering, path length, modularity, can facilitate the identification of organizational patterns in a large-scale complex brain networks (McIntosh and Korostil, 2008).

Numerous investigations over the last two decades have been conducted regarding motor expertise (e.g., Chang, 2014; Debarnot et al., 2014; Herholz and Zatorre, 2012; Jancke, 2009; Jancke et al., 2009; Yang, 2015) but inconsistencies across findings were observed. Just to name a few, when compared to non-experts, fMRI studies have found, in experts, reduced brain activity within task-relevant areas during the execution of motor-related tasks (Petrini et al., 2011, Haslinger et al., 2004) whereas others have shown the reversed pattern (Calvo-Merino et al., 2005; Kim et al., 2011). Along the same lines, studies by Han et al. (2009) have reported greater FA values in experts whereas Imfeld et al. (2009) have revealed lower FA values. As for Schmithorst and Wilke (2002) and Bengtsson et al. (2005), they have found a combination of greater and lower values in expert performers. To unravel these discrepancies, we conducted a novel literature review. Aware that sample constitution, characteristics of the practiced motor tasks, hours spent in training, measurement tools, and analyses used by researchers could all lead to bias, we adopted strict inclusion criteria.

First, specific attention has been paid to the literature from the past fifteen years. Articles were found with the Pubmed and ScienceDirect data bases. The keywords were motor expertise, cortical changes, motor training, healthy subjects, sMRI and fMRI.

Second, motor learning literature examining cortical changes over the course of learning was not considered because of the small amount of time spent in motor training compared to the sheer volume of hours practiced to achieve expertise. Research examining cortical changes induced by motor training has employed two approaches: cross-sectional and longitudinal. In a cross-sectional approach, groups of individuals possessing different motor skill levels are compared and researchers attempt to identify differences in their structural and/or functional cortical organization. In the majority of cases, the brains in motor experts have been examined and compared with those in untrained sedentary subjects at a single time point. As stated by Bezzola et al. (2012), these studies reveal the effects of the late stages of motor learning on cortical organization. Alternatively, to examine the time course of cortical changes, longitudinal designs were employed in which healthy individuals were exposed to specific motor training interventions lasting several hours spread over a week or several months. These designs involved repeated measures of cortical parameters throughout the intervention namely prior to the intervention onset, during the intervention, and after the intervention. Findings from these studies have mainly enabled cortical changes induced during the early stages of motor learning to be revealed (Bezzola et al., 2012). Consequently, only cross-sectional studies which have focused on cortical differences between motor expert performers and non motor expert performers were considered.

Third, different forms of work requiring motor expertise, such as sport and music, were considered only if the size of the samples was greater than or equal to 10 and if expert and non expert group sizes were equal. Unequal sample sizes result in confounding whereas a small sample size does not enable statistical calculations to be accurate and 
reliable which subsequently is responsible for misinterpretations of study findings. Participants should also be healthy adults.

Fourth, since cortical changes depend on the types of movements executed by the subjects (Filippi et al., 2010; Jensen et al., 2005; Tyc and Boyadjian, 2006; Voelcker-Rehage and Niemann, 2013), only coordinative tasks were considered (e.g., balance, eye-hand coordination, leg-arm coordination, and reaction to moving objects and people; see Voelcker-Rehage et al.'s classification, 2013). Consequently, cardiovascular, strength tasks, endurance, and reaction time tasks were excluded from this review.

Fifth, focus was put on studies using novel brain imaging technologies such as sMRI (e.g., VBM, Diffusion MRI) and fMRI. Consequently, studies investigating practice-related changes and using tools such as transcranial magnetic stimulation, electroencephalography, and magnetoencephalography were excluded. This criterion was chosen to ensure that studies included in this review would share accurate spatial resolution (Yang, 2015). Regarding fMRI exams, as we investigated the effect of prolonged and intensive motor training on the brain, studies using motor tasks directly related to those performed daily in training were included in this review. Consequently, studies employing motorrelated tasks such as imagery, observation, anticipation or motor planning were not considered. Task-free fMRI studies were also taken into account as there is a close match in functional brain architecture during rest and task performance (Cole et al., 2014; Lewis et al., 2009; Smith et al., 2009).

Sixth, only studies using whole brain analysis methods were selected. It is of utmost interest to get information on the whole brain as it is unlikely that a single area should be working on its own. Moreover, some teams arrived at conflicting results depending on the analysis performed: ROI and whole brain analyses (e.g., Hufner et al., 2011).

Finally, studies investigating changes in cortical networks induced by intensive and extensive motor training were taken into account. To the best of our knowledge, such studies have rarely been included in motor expertise reviews.

This literature review thus summarizes and discusses research investigating structural and functional changes induced by an intensive and an extended period of motor training in music and sport. Alterations are documented on a local level as well as a network level. More specifically, one can wonder how brain structures and functions differ in motor expert performers and non-experts. Regardless of the nature of changes, be they structural or functional, are these changes reflected by higher or lower values in expert brains compared to nonexperts? Where in the brain do these changes occur? And, do cortical changes take place in similar and/or distinct localizations for different kinds of motor expertise?

\section{Which attributes differentiate motor experts from non-experts? a local approach}

When examining the literature related to structural and functional cortical changes associated with motor expertise, 13 out of the 23 reviewed studies focus on changes on a local level (see Table 1). Data are analyzed in a way where each area has been considered separately without taking into account relationships with other areas. Among these 13 studies, 11 focused on structural changes on a local level which have been documented in cortical thickness, in gray and white matter architecture. Among the remaining studies, which are of a functional nature, two investigated local activation. In this review, according to our third inclusion criterion, motor experts are from all walks of life ranging from music to sports. As the scientific community recognizes that motor training shapes the human brain in areas related to the execution of the task at hand, it is not altogether surprising to observe different areas at work when practicing multifarious motor disciplines (see Box 2). To identify common principles of brain changes induced by lengthy-extensive motor training, each identified area was ordered in the system it belongs to. Systems are identified on the basis of works by
He et al. (2009) and Wang et al. (2013) who investigated the modularity of human brain networks stemming from resting-state BOLD signals. Thus, five well known systems that are the sensorimotor/auditory, attention, visual, default mode, and limbic/subcortical systems are considered in this section.

\section{Box 2}

Different types of motor disciplines and their requirements.

In the musical domain, playing an instrument requires translating visually perceived musical symbols into complex sequential digital movements and checking auditory output concomitantly. Consequently, auditory, motor and visual systems are enrolled (Zatorre et al., 2007). Moreover, expert musicians usually play in an ensemble where they have to interact with each other to produce a coherent and cohesive musical piece. This requires them to take visual cues from a conductor or from each other and to listen, at the same time, to their own part as well as to parts played by others which leads to involve and develop their attentional system (Keller, 2001). It has also been shown that changes in brain structure varied between musicians based on the instrument they played. Bangert and Schlaug (2006) showed that pianists' and violonists' brains are anatomically different and more specifically in the precentral gyrus associated with hand movement representation (Omega Sign). Pianists displayed a more prominent omega sign in the left hemisphere than in the right hemisphere whereas violonists exhibited a prominent omega sign only in the right hemisphere. In the sport domain, two subcategories are also identified according to the stability of the environment: closed and open/perceptually driven skills. Closed skills, in which the environment is highly consistent and predictable, depends to a great extent on form for their successful executions (e.g., dance, gymnastics). These skills are initiated by a performer (self-initiated movements). In contrast, open skills, in which athletes must react in a dynamically changing and unpredictable environment, rely heavily on perception for their successful executions (e.g., fighting sports: judo, badminton; team sports: basket-ball, football) (Magill, 2010). These skills are referred to as externally triggered skills since they are imposed on a performer by some external agents. Consequently, performing open-skill sports entails high levels of visual attention, fast and flexible decision making (Magill, 2010). This form of practice contrasts with the execution of closed skill sports which requires a precise body segment organization combined with proprioceptive sensations and multisensory feedback as these skills strongly rely on form for their successful execution (Magill, 2010). Consequently, it is not altogether surprising to observe different areas at work when practicing these diverse motor skills. Moreover, it has been shown that self-initiated skills do not involve the same cortical areas/pathways as externally triggered skills, though there may be some overlaps (Goldberg, 1985; Gowen and Miall, 2007; Kuruma et al., 2007; van Donkelaar et al., 1999). For instance, Kuruma et al. (2007) showed that an internal loop, constituted of the SMA and basal ganglia, was preferentially at play when performing a self-initiated movement. Conversely, during the execution of an externally triggered movement, an external loop, composed of the parietal and lateral premotor cortices, was dominant. Along the same line, differences in the involvement of cortical areas have also been found when performing self-initiated vs externally triggered skills (Gowen and Miall, 2007; Hoshi and Tanji, 2006; Jenkins et al., 2000; Rao et al., 1993). Gowen and Miall (2007) revealed that compared to externally triggered movements, self-initiated movements elicited greater activation within the right cerebellar crus I, pre-SMA, dorsal premotor cortex, right superior parietal/precuneus and left precuneus. 
Table 1

Synthesis of cross-sectional studies investigating changes induced by extensive and intensive motor training. Characteristics of the subjects, measures and techniques used in these studies are provided.

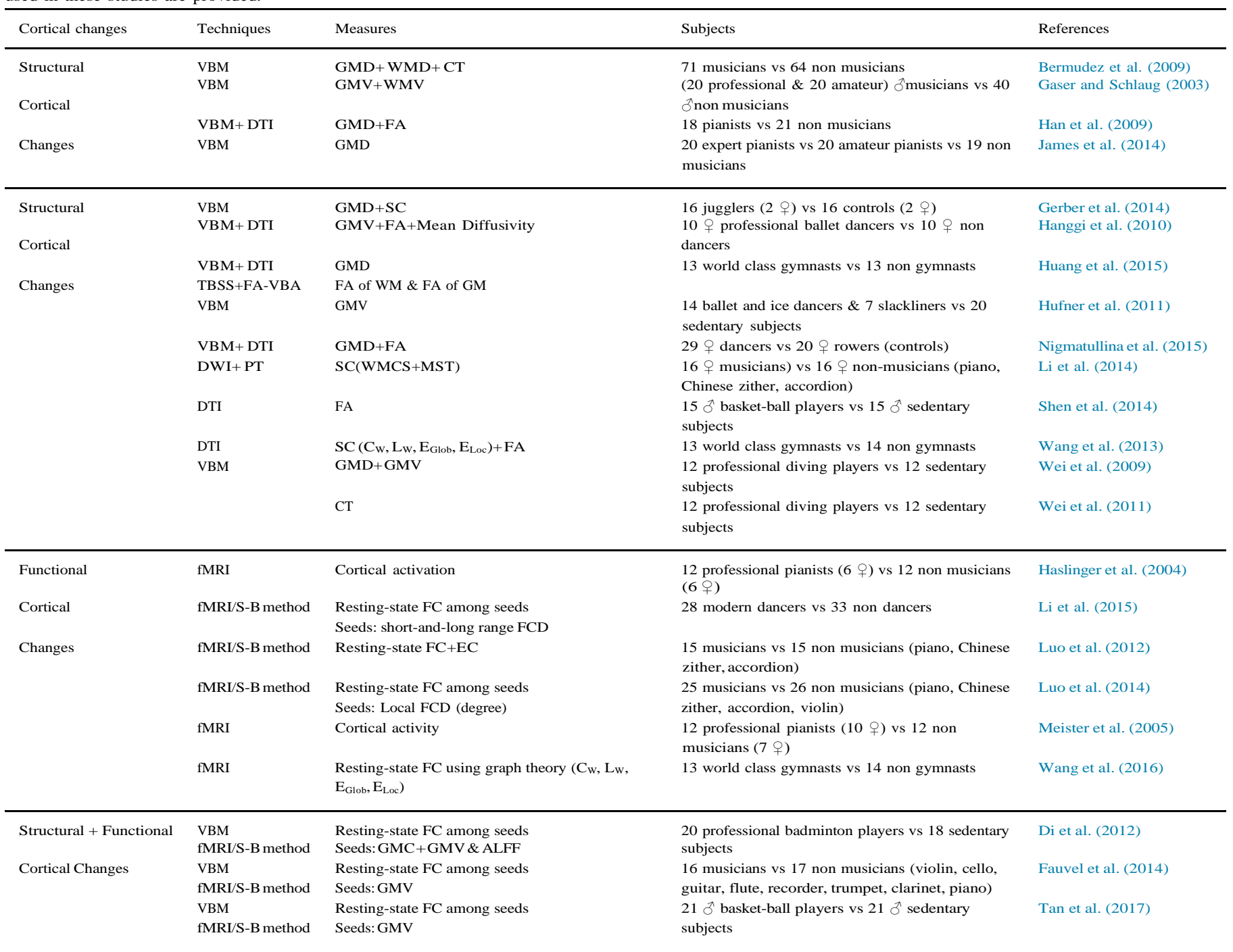

List of abbreviations: ALFF = Amplitudes of Low Frequency Fluctuations, CA = Cortical Activation, CT = Cortical Thickness, $\mathrm{C}_{\mathrm{W}}=\mathrm{Weighted}$ Clustering coefficient, DTI $=$ Diffusor Tensor Imaging, DWI $=$ Diffusion-Weighted Imaging, E $_{\text {Glob }}=$ global Efficiency, E $_{\text {Loc }}=$ local Efficiency, EC $=$ Effective Connectivity, FA $=$ Fractional Anisotropy, FA-VBA=Voxel-Based Analysis of Fractional Anisotropy, FC = Functional Connectivity, FCD = Functional Connectivity Density, GMD= Gray Matter Density, GMV = Gray Matter Volume, $\mathrm{L}_{\mathrm{w}}=$ characteristic path Length, $\mathrm{MEG}=$ MagnetoEncephaloGraphy, MST =Minimum Spanning Tree, $\mathrm{PT}=\mathrm{Probabilistic}$ Tractography, ROI=Regions of Interest, SC=Structural Connectivity, S-B method=Seed-Based method, TBSS =Tract-Based Spatial Statistics, VBM=VoxelBased Morphometry, WMCS= White Matter Connectivity Strength, WMD=White Matter Density, WMV= White Matter Volume.

Omega sign: anatomical feature of the precentral gyrus.

Note. When gender is not mentioned in the subject column, the population is composed of both genders.

\subsection{Investigating cortical thickness changes induced by long and intensive motor training}

Two studies, one related to sport (Wei et al., 2011) and the other to music (Bermudez et al., 2009) have investigated the relationship between motor expertise and cortical thickness. Wei et al. (2011) recruited diving players whereas Bermudez et al. (2009) selected musicians without specifying their speciality instrument. Results of both teams are in agreement as higher values of cortical thickness were observed in experts compared to non-experts. However, areas in which these superior values have been detected are distinct in music and sport: the planum temporal and frontal gyrus in its middle part and inferior parts in musicians (Bermudez et al., 2009) versus the left superior temporal sulcus, right orbitofrontal cortex, and right parahippocampal gyrus in diving players (Wei et al., 2011). More generally, when referring to the systems areas belong to, we found both common and distinct systems enrolled in these two motor disciplines. Greater cortical thickness is identified: (i) in music and sport experts within attention system, (ii) in musicians within auditory system, and (iii) in elite athletes within visual and limbic/subcortical systems. Involvement of the attention system both in proficient musicians and diving players is not surprising when analyzing the characteristics of their practice. Both disciplines require motor experts to take into account a vast amount of information and to select, very quickly, relevant pieces of information that are useful to the execution of the task at hand in order to produce a subsequent successful action (see Box 1). Information is mainly of auditory nature for musicians and of visual and proprioceptive nature for athletes. Conclusions from these analyses must be interpreted with great caution as they drew from only two studies. 


\subsection{Investigating gray matter changes induced by long and intensive motor training}

When comparing non-experts to motor experts practicing music, key-board/string instruments, piano, badminton, gymnastics, dance/ slackline, diving, and juggling, discrepancies in terms of direction alterations are detected for gray matter indices. Some research teams found higher gray matter indices in motor experts (Bermudez et al. 2009; Di et al. 2012; Fauvel et al. 2014; Gaser and Schlaug 2003; Gerber et al. 2014; Huang et al. 2015; Hufner et al. 2011) whereas one team observed lower values (Hanggi et al. 2010). Lastly, others reported a combination of greater and weaker gray matter values in skilled samples (Han et al. 2009; James et al. 2014; Nigmatullina et al. 2015; Wei et al. 2009) (see Supplementary Table 2).

First, when focusing on systems exhibiting higher gray matter indices in experts compared to non-experts (Bermudez et al., 2009; Di et al. 2012; Fauvel et al. 2014; Gaser and Schlaug 2003; Gerber et al. 2014; Huang et al. 2015; Hufner et al. 2011), we find that the sensorimotor, attention, and limbic/subcortical systems are common to musicians and athletes whereas other systems are specific to each of these populations. A specific system found exclusively in musicians is the auditory system in all the 3 reviewed musical studies (Bermudez et al. 2009; Fauvel et al. 2014; Gaser and Schlaug, 2003) and a system solely seen in athletes is the visual system $75 \%$ of the sport studies (Gerber et al. 2014; Huang et al. 2015; Hufner et al. 2011). Interestingly, in 50\% of the studies (Di et al. 2012; Gaser and Schlaug 2003; Hufner et al. 2011), subcortical alterations have been observed in the cerebellum, an area known to be involved in motor timing and execution (Mauk et al. 2000), in prediction of sensory consequences of movements and detection of errors in these predictions (Hardwick et al. 2013; Penhume and Steele 2012) and in control of oculomotor function, grip forces and voluntary limb movement (Manto et al. 2012). Finding alterations within the cerebellum among motor experts be it in badminton, music, or dance/slackline seems to make sense as all the aforementioned cognitive functions are involved when playing a piece of music as well as when performing a sport action.

Second, when focusing on systems exhibiting weaker gray matter indices in experts compared to non-experts, only one study has been found (Hanggi et al. 2010). In the cortex of professional female ballet dancers, this research team observed lower values within the sensorimotor, default mode, and subcortical systems which could be interpreted as markers of great expertise (see Supplementary Table 2). Interestingly, sensorimotor areas (premotor cortex and SMA) and subcortical structure (putamen) are recognized to be involved in motor control processes (Brown et al. 2006).

Third, a combination of increase and decrease patterns has also been observed in the musical and sport domains when comparing motor expert performers to non-experts (see Supplementary Table 2). In professional pianists, dancers and divers, sensorimotor, attention and visual areas exhibit lower as well as greater gray matter values (Han et al. 2009; James et al. 2014; Nigmatullina et al. 2015; Wei et al. 2009). The same holds true for the cerebellum. Weaker gray matter values within the anterior sensorimotor and posterior emotional cerebellar zones are observed in athletes (Nigmatullina et al. 2015; Wei et al. 2009) while in professional pianists, Han et al. (2009) and James et al. (2014) detected greater values within the posterior cognitive cerebellar zone, a zone known for its involvement in higher-order cognitive processing (O'Reilly et al. 2010; Stoodley and Schmahmann 2009) (See Supplementary Table 2).

To account for these divergent gray matter findings, we can provide two possible explanations. The first one is related to a number of methodological issues: characteristics of the tasks, sample constitution, and markers used to examine the structural gray matter architecture. The second is based on the expansion-renormalization model (Reed et al. 2011; Wenger et al., 2017a).

\subsubsection{Methodological issues}

A first suggestion is related to the characteristics of the tasks. As proposed by Hanggi et al. (2010), gray matter changes may be the results of the intensive and extensive practice of a particular task whereas another task may generate no changes. This suggestion does not fit well with the reviewed findings. Task-related differences were expected when comparing skills that have little in common like badminton and piano playing (e.g., Di et al. 2012 vs James et al. 2014) or badminton and dance (e.g., Di et al. 2012 vs Hanggi et al. 2010). However, it is problematic to see different patterns when experts are compared to nonexperts in similar activities. In ballet dancers, two patterns are found: lower gray matter values (Hanggi et al. 2010), and a combina- tion of greater and weaker values (Nigmatullina et al. 2015). In musi- cians, two patterns are also revealed: greater values (Bermudez et al. 2009, Fauvel et al. 2014, Gaser and Schlaug 2003), and a combination of greater and weaker values (Han et al. 2009, James et al. 2014) (see Supplementary Table 2). Clearly, there are more than task-related influences at play to explain these discrepancies.

Sample constitution and more specifically participants' gender, experts' selection, experts' experiences, and match between control subjects and expert cohorts is a second suggestion to explain the divergent results. When considering participant gender, it has been shown that gray matter indices within temporal area are greater in females than males (Good et al. 2001; Sowell et al. 2007; Witelson et al. 1995). In dance, groups are composed of exclusively female subjects (Hanggi et al. 2010; Nigmatullina et al. 2015) whereas in music, groups include only male subjects (Gaser and Schlaug 2003) or both genders (Bermudez et al. 2009; Fauvel et al. 2014; Han et al. 2009; James et al. 2014) (see Table 1). The lack of homogeneity in expert sample constitution can also account for the observed divergences. Bangert and Schlaug (2006) showed that changes in brain structure varied between musicians based on the instrument they played (see Box 2). In their studies, Gaser and Schlaug (2003) recruited keyboardists and string players; Fauvel et al. (2014) selected violin, guitar, flute, recorder, trumpet, clarinet and piano players whereas Han et al. (2009) and James et al. (2014) only enrolled pianists. Bermudez et al. (2009) selected musicians without providing information on their speciality instrument. In sport, Hanggi et al. (2010) and Nigmatullina et al. (2015) enlisted ballet dancers whereas Hufner et al. (2011) recruited a mixed bag of subjects: ballet dancers, ice dancers, and slackliners. This is further complicated by heterogeneous features of experts' experiences such as duration of practice and number of hours of training per week. For instance, in dance, duration of practice and weekly training hours vary a great deal: 14.2 years with $35.8 \mathrm{~h}$ per week (Hanggi et al. 2010), 16 years with $8.8 \mathrm{~h}$ per week (Nigmatullina et al. 2015), 16.92 years for dancing and 2.79 years for slacklining with $11.75 \mathrm{~h}$ per week (Hufner et al. 2011). The same observation holds true for music: 16.9 years with $14.7 \mathrm{~h}$ per week (Bermudez et al. 2009), 16 years with several times per week (Fauvel et al. 2014), 17.7 years with at least $10.5 \mathrm{~h}$ per week (Gaser and Schlaug 2003), 10.4 years with number of hours per week, not provided (Han et al. 2009), 18.3 years with $30 \mathrm{~h}$ per week (James et al. 2014).

The control subjects' selection could also clarify the inconsistencies in terms of direction alterations. Though controls are supposed to be matched in gender-and age with motor expert performers, in some studies, controls possess a certain amount of motor expertise as they practiced: (i) music for less than 3 years (Bermudez et al. 2009), (ii) leisure sports such as ballet dance and ice dance for two participants (Hufner et al. 2011), and (iii) rowing (Nigmatullina et al. 2015). The fact that experts and controls share some motor experiences could throw findings into doubt as motor experience is the factor under investigation. On the opposite, Hanggi et al. (2010) in ballet dance, Gaser and Schlaug (2003), Han et al. (2009), James et al. (2014) in music selected their control subjects perfectly well. These had never experienced musical training or dance, figure skating, gymnastics, synchronized swimming, equestrian vaulting training and had never performed 
any competitive sports. All things considered, as of today, no data are available to unravel the different weight of these different factors on gray matter alterations.

Inconsistencies in terms of gray matter alterations may be due to the markers used to examine the structural gray matter architecture i.e. either gray matter concentration/density or gray matter volume. These two indexes represent different metrics and hence could lead to different results. As an example, Di et al. (2012) and Wei et al. (2009) used both metrics on the same populations and found distinct results. The former revealed higher gray matter concentration/density within the cerebellum in badminton players when compared to non-experts and no gray matter volume differences between the two groups. The latter showed higher and lower gray matter density for expert performers and no significant difference in gray matter volume between experts and non-experts.

\subsubsection{Expansion-renormalization model}

Inconsistencies in terms of gray matter direction alterations within various brain areas may also be explained by the expansion-renormalization model. In the present review, discrepancies are observed both within and between studies. Within studies, practice-related changes are reflected by the coexistence of increased and decreased gray matter in various brain areas in motor experts practicing the piano, dance, or diving (Han et al. 2009; James et al. 2014; Nigmatullina et al. 2015; Wei et al. 2009). In studies which investigated gray matter changes in similar activities, practice-related changes are characterized by different patterns. In professional dancers, we observe a decrease pattern (Hanggi et al. 2010) and an increased and decreased combination pattern (Nigmatullina et al. 2015) whereas in musicians, we see an increase pattern (Bermudez et al. 2009; Fauvel et al. 2014; Gaser and Schlaug 2003) and an increased and decreased combination pattern (Han et al. 2009; James et al. 2014) (See Supplementary Table 2). The expansion-renormalization model which could explain the aforementioned inconsistencies is based on animal models and theoretical accounts of skill acquisition and development (e.g., Reed et al. 2011; Wenger et al., 2017a; Xu et al. 2009). It posits that during skill acquisition, human brain structure changes and follows sequences of expansion, selection, and renormalization (Fu and Zuo 2011; Makino et al. 2016). In the early stages of learning, brain matter volume increases in task-relevant areas which means that the number of brain cells, such as neurons and glia cells, raises. Then, the number of these cells decreases over time as a selection process operates. This selection is based on the principle that the most frequently used cells are kept whereas the ones that are no longer needed are pruned away. When the selection process has ended, brain matter volume is nearly back to its initial size whilst skill performance stays high or still carries on increasing. Furthermore, Wenger et al. (2017b) have observed this pattern of expansion-renormalization in gray matter volume within the right primary motor cortex in right-handed subjects during learning of fine motor skills of writing and drawing with their non-dominant left hand. They also found a trend for renormalization within the left primary motor cortex and the right putamen with threshold levels of permissive significance (uncorrected, without FWE-corrected cluster extent threshold). These findings lead them to suggest that different regions could follow the same expansion-renormalization pattern during motor training but at a different pace. This could explain the combination of gray matter increase and decrease patterns in task-relevant areas/systems observed within studies (Han et al. 2009, James et al.2014, Nigmatullina et al. 2015) as well as the different patterns seen across studies (see Supplementary Table 2). Besides, the point in time when the MRI measures are taken is of great importance and even could account for gray matter discrepancies in terms of direction alterations observed in the reviewed studies. If the measures are collected after the gray matter expansion stage, one can expect to observe a decrease. Conversely, during the expansion stage, an increase can be expected. At present time, this point is beyond the control of the experimenter.

Most importantly, at present time, there are only speculations to explain the cellular and molecular mechanisms underlying structural measures obtained with VBM. A gray matter alteration could be due to neurogenesis, angiogenesis, gliogenesis, synaptogenesis and changes in neuronal morphology (for a review, see Zatorre et al. 2012). This alteration is likely never the consequence of a unique mechanism occurring autonomously but presumably implies a combination of several mechanisms (Zatorre et al. 2012). Developing histological studies and MRI imaging in animals (e.g., Keifer et al. 2015; Lerch et al. 2011) is a necessary stage to deepen our comprehension on micro-level changes underlying VBM signals and may help understand discrepancies observed in gray matter direction alterations induced by extensive and intensive motor training.

\subsection{Investigating fractional anisotropy (FA) changes induced by long and intensive motor training}

In the musical and sport domains, some research teams found higher FA values in motor expert performers when compared to non-experts (Han et al. 2009; Shen et al. 2014) whereas others registered weaker values (Hanggi et al. 2010; Huang et al. 2015) (see Supplementary Table 3).

When focusing on areas exhibiting higher FA values in motor experts compared to non-experts, (Han et al. 2009: music with pianists versus Shen et al. 2014: basket-ball), we find common and distinct systems enrolled in these two motor disciplines. Greater FA is seen: (i) in pianists and basket-ball players within attention and subcortical systems, and (ii) in basket-ball players only within the sensorimotor, visual, and default mode systems (see Supplementary Table 3).

When focusing on systems exhibiting lower FA values in sport experts than those registered in controls (Hanggi et al. 2010: in ballet dance; Huang et al. 2015: in gymnastics), two common systems that are the sensorimotor and attention systems are found in both studies whereas the visual system is only registered in Huang et al. (2015) study. Finding common systems is not surprising since both disciplines are closed and self-initiated activities which share similar characteristics such as precise motor control, balance, coordination, flexibility, power, developed proprioceptive sensations, and focused attention during the execution of movements. However, that weaker FA values within visual system are only detected in gymnasts is puzzling. Compared to other reviewed studies, the participants recruited by Huang et al. (2015) possess the highest standard of motor expertise which could account for this observation as all the participants won gold medals in the Gymnastic World Championships or the Olympic Games. Weaker FA within the visual system could be a marker of outstanding motor expertise for closed and self-initiated activities. However, some additional investigation is necessary to support this suggestion.

To account for these divergent diffusion tensor imaging (DTI) findings, two explanations are suggested: differences in experts' experiences and cellular and molecular mechanisms.

First, as suggested by Paul and Cnossen (2018), heterogeneous changes could be attributed to the amount of time spent practicing. In the present review, the initial onset of training and the amount of practice vary a great deal between the four aforementioned studies though the subjects of these studies are all motor experts but at different degrees. Indeed, pianists of both genders selected by Han et al. (2009) commenced training at 12 years of age whereas male basket-ball players recruited by Shen et al. (2014) started training at 13 years of age and practiced this activity moderately ( $7 \mathrm{~h}$ per week). The pianists had been practicing for 10.4 years and the basket-ball players for 6 years and a half. As for Hanggi et al. (2010) and Huang et al. (2015), they recruited female professional ballet dancers and world class gymnasts of both genders. The dancers started practicing at 7 years of age whereas the gymnasts began training at 4.5 with a weekly practice of $36 \mathrm{~h}$ for both populations. Dancers and gymnasts had been training 
respectively for 14 years and 16 years by the time of the experiment. Thus, we propose that a 'moderate' practice, as in studies by Han et al. (2009) and by Shen et al. (2014), lead to increases in FA whereas practicing a motor activity in an elite context, as in studies by Hanggi et al. (2010) and Huang et al. (2015), could result in decreases in FA. Of course, this suggestion is pure speculation and warrants further investigation.

Findings from the literature investigating FA changes over the time course of motor learning could be of help to explain discrepancies observed in the present review. To our best of knowledge, only three studies have investigated this issue (Landi et al. 2011; Scholz et al. 2009; Taubert et al. 2010). In these longitudinal studies, individuals went through specific motor training interventions such as practicing a juggling task during $15 \mathrm{~h}$ spread over 30 days (Scholz et al. 2009) or a whole-body balancing task during $4 \mathrm{~h}$ and $30 \mathrm{~min}$ spread over 42 days (Taubert et al. 2010) or a visuomotor adaptation task during $5 \mathrm{~h}$ and 15 min spread over 7 days (Landi et al. 2011). They underwent scanning at the onset and at the end of training (Landi et al. 2011; Scholz et al. 2009) and also during training (Taubert et al. 2010). These studies reported greater but also weaker FA values within parietal and frontal white matter areas at the end of motor training compared to values observed at the training onset: greater values for Scholz et al. (2009) and Landi et al. (2011) and weaker values for Taubert et al. (2010). Moreover, Taubert et al. (2010) described how FA data evolved across training sessions. They found transient changes within the dorsolateral prefrontal and precentral white matter regions as well as changes evolving slowly and bilaterally within the dorsal prefrontal area but in different parts than the ones mentioned above. Taken together, motor learning studies reported FA changes that displayed opposite patterns as well as changes occurring at different paces within different areas. These different and coexisting patterns of changes, apparently discordant, could be explained by the fact that different regions may follow the same change patterns but at different rates. This suggestion would strengthen the expansion-renormalization model (Reed et al. 2011; Wenger et al., 2017a). However, this proposition should be taken with a grain of salt. First, to our knowledge, no studies have shown that FA changes in white matter elicited by motor learning/training follow an inverse quadratic function i.e. an initial expansion followed by a renormalization. Second, comparing cortical changes induced by motor learning/training lasting several hours to $100 \mathrm{~h}$ at most with those resulting from training where individuals are exposed to approximately ten thousand hours to achieve motor expertise is not adequate. Underlying change mechanisms might be different in these two situations (Bezzola et al., 2012).

Second, cellular and molecular mechanisms could help understand discrepancies detected in FA changes of directions. DTI measures are known to be receptive to tissue properties such as variation in myelin, axon diameter, packing density of fibers, axon permeability, and fiber geometry (Beaulieu 2009; Concha et al. 2010; Takahashi et al., 2002; Wedeen et al. 2005). Hanggi et al. (2010) and Huang et al. (2015) hypothesized that FA reduced values observed in expert gymnasts and dancers compared to those detected in control individuals are caused by increases in axonal diameter. Hanggi et al. (2010) added other hypotheses such as increase in membrane permeability for water molecules and presence of crossing fibers within a voxel. As evidenced by this literature, multifarious micro mechanisms could account for FA changes. So far, no one is able to disentangle the specific contributions of these mechanisms via DTI alone (Concha, 2014; Zatorre et al. 2012). Besides, as over two-thirds of the voxels in a standard brain data set comprise crossing fibers (Jeurissen et al. 2013), using DTI is inappropriate since DTI enables assessment of tissue microstructure in cortical areas with only a single fiber orientation (Tuch et al. 2002). This implies that DTI measures need to be interpreted with extreme caution. To solve these issues, diffusion spectrum imaging, which is able to take into account several fiber directions within a voxel (Tuch 2004), should be employed. Research teams should undergo histological and MRI studies on animals. These proposals, if applied, would provide a more precise description of white matter tissue characteristics and their development.

\subsection{Investigating cortical activation changes induced by long and intensive motor training}

Haslinger et al. (2004) and Meister et al. (2005) examined cortical activation during the execution of hand movements in professional pianists practicing for at least 16 years and in controls. They instructed the subjects to perform, during scanning, simple and complex finger tapping tasks; at a $1 \mathrm{~Hz}$ rate with the index, middle, ring and little fingers of both hands for Haslinger et al. (2004) and with all the fingers of the right hand for Meister et al. (2005). Both research groups reported weaker cortical activity in professional pianists as compared to musically naive subjects within similar motor areas, i.e. the dorsal premotor cortex and SMA proper. Moreover, Haslinger et al. (2004) found greater cortical activity in musically naive controls within additional areas not detected in the study of Meister et al. (2005) such as the cerebellum, prefrontal cortex, insula, and basal ganglia. To account for this additional activity, several suggestions are made: (i) different characteristics of the tasks performed during scanning, (ii) gender mismatch between pianists and controls in Meister et al. (10 female subjects \& 2 male subjects for pianists vs 7 female subjects \& 5 male subjects for controls), and (iii) gender mismatch within the pianist group in Meister et al. (10 female subjects vs 2 male subjects). Considering the last decade's findings that have reported differences in functional connectivity and network topology between women and men in various cognitive tasks (Douw et al. 2011; Gootjes et al. 2006; Pavlova et al., 2015; Tomasi and Volkow 2012; Zhang et al. 2018), it seems methodologically justified to match genders between and within groups

Another point to consider is that most of the time, an activation decrease pattern recorded in motor performers has been interpreted as a gain in neural efficiency. Less investment of neural energy is required after intensive and extensive motor training. According to Poldrack (2015), the term 'efficiency' is vacuous and simply redescribes the process of activation reduction without providing any insight about the mechanisms at play. To achieve a better understanding of activation reduction in motor expert brains compared to those of non-experts, it would be necessary to examine whether these two kinds of populations perform the same neuronal computations and whether the energy expenditure is the same (Poldrack 2015). Appraising these similarities is a challenge as it involves supporting the null hypothesis which is never proven true. As explained by Poldrack (2015) and based on Logan's theory of automatization (1988), as expertise develops in a particular domain, individuals process information differently (e.g., Grainger et al. 2012; Poldrack et al. 1998). They switch from an algorithm-based processing to a memory-based processing. Consequently, speaking of efficiency does not make any sense since each group executes a different task. Moreover, supposing that each group uses the same neural computation in terms of quality, time, and intensity, they could still differ in terms of metabolic expenditure. Using deoxyglucose uptake and single-neuron recording, Picard et al. (2013) were the first to show a broad change in the relation between metabolic activity and neuron activity in monkeys they trained for 1-6 years to perform an internally generated motor task. This alteration translates into a reduction of metabolic activity within the primary motor cortex when performing a highly skilled task (i.e., internally generated motor task) compared to an untrained motor task (i.e., visually guided task) and into no changes in measures of neuron firing between these two tasks. This finding suggests a difference in energy usage in apparently identical neuronal computations. For obvious reasons, the methodological approach used by Picard et al. (2013) is unenforceable in human subjects. Thus, as recommended by Picard et al. (2013), interpretation of functional imaging results should be exercised with great caution, more 
specifically when becoming a motor expert performer after spending enormous amount of time in practice.

Based on Picard et al. (2013) recommendation in interpreting fMRI results, methods used in motor learning studies, such as multi-voxel pattern analysis (MVPA; Swisher et al. 2010; Freeman et al. 2011; Wiestler and Diedrichsen, 2013) or time-evolving communities detection (Bassett et al., 2013), seem promising to investigate cortical functional changes induced by extensive motor training of at least several thousand hours. MVPA, recognized to be able to identify differences in voxel-by-voxel activity pattern within a particular cortical area, has been employed by Wiestler and Diedrichsen (2013). They have found that motor skill training generated particular changes in the cortical representations of motor sequences. Activity patterns of practiced digital sequences were more easily discernible from each other compared to unpracticed sequences. In other words, each trained motor sequence was associated with a specific and distinct cortical activation pattern especially in the supplementary motor area and this, without generating any average increments in brain activity.

As for Bassett et al. (2013), they developed algorithms to extract time-evolving communities from patterns of brain activity during motor learning. They revealed the presence of two kinds of connected nodes: a temporal core and a periphery. The temporal code is composed of densely connected nodes and encompasses sensorimotor and visual areas whose connectivity does not vary a great deal over the course of motor learning. Conversely, the periphery is made up of sparsely connected nodes and comprises multimodal association areas whose connectivity is frequently altered. Separation between core and periphery varies across learning and is a good indicator to predict successful performance.

\subsection{From a local approach to a network approach}

This literature review shows on a local level that brain structures and functions differ in motor expert performers and non-experts. Congruent with the scientific literature, localization of cortical alterations resulting from a lengthy and extensive training is closely related to the characteristics of the practiced motor task (Buschkuehl et al. 2012; Doyon et al. 2002; Guida et al. 2012; Jueptner et al. 1997). Consequently, observing, among experts from all walks of life, practice-related changes which happened in different areas/systems is not surprising. However, one has to keep in mind that two different motor disciplines which appear dissimilar could share common cortical systems among different expert cohorts as these disciplines feature similar intrinsic properties. This is what has been revealed in this review. We have observed that practice-related changes occur in common systems shared by musicians and athletes but also in specific systems for each of these populations. To summarize, compared to non-experts, greater cortical thickness, FA, and gray matter are detected in the attention system both in musicians and athletes. On the other hand, greater cortical thickness and gray matter are exclusively registered within the auditory system in musicians whereas higher cortical thickness, FA, and gray matter are solely found within the visual system in athletes. Conversely, compared to non-experts, weaker FA is observed within common systems shared by both dancers and gymnasts: the sensorimotor and attention systems (see Supplementary Tables 2 and 3).

As for the direction changes and irrespective of the cortical indices employed in the reviewed studies, we find three patterns of direction changes: increase, decrease, and a combination of increase and decrease (see above and Supplementary Tables 2 and 3). More specifically, a consensus seems to have been reached on the direction of alterations in cortical thickness (increase) and in cortical activation (decrease) but discrepancies in gray matter indices and FA still remain. Some research teams found higher gray matter indices in motor experts when compared to non-experts; one team observed lower values; and others reported a combination of greater and weaker gray matter values in skilled samples. Lastly, when considering FA, some research teams found higher values in motor experts whereas others registered weaker values.

In view of the aforementioned findings, interpreting the coexistence of increase and decrease of diverse cortical indices within various brain areas/systems challenges our understanding of the underlying neuronal mechanisms of motor expertise. Hence, conducting analyses on a network level seems a promising method in order to deepen our understanding of the mechanisms underpinning cortical changes. This is a particularly important point as the brain is far from being a set of disconnected cortical areas. In reality, to enable cognitive functions or to acquire/perform motor tasks, cortical areas communicate with each other in complex network patterns. When examining the literature related to structural and functional cortical changes associated with motor expertise, 7 out of 23 reviewed studies focus on changes on a network level by considering interactions between pairs of brain components (Di et al. 2012; Fauvel et al. 2014; Gerber et al., 2014; Li et al. 2015; Luo et al. 2012, 2014; Tan et al. 2017). Among these studies, only Gerber et al. analyze structural changes (see Table 1).

\section{Which attributes differentiate motor experts from non-experts: A network approach}

\subsection{Investigating structural and functional changes in cortical networks: $A$ pair-wise interaction approach}

When considering structural connectivity, Gerber et al. (2014) conducted a connectivity analysis to investigate whether expert jugglers possessed a specific brain anatomy induced by juggling training and related to the characteristics of the practiced motor task. They examined gray matter connectivity between the visual areas (hMT/V5, lingual gyrus) and the intraparietal sulcus (IPS): an area recognized to be involved in the integration of multimodal sensory information when planning and executing complex movements. Interestingly, although expert jugglers displayed higher gray matter density than non-jugglers within visual and IPS areas, no difference between both groups was found in terms of structural connectivity between these areas. In our opinion, the huge heterogeneity in jugglers' experiences (i.e., number of training hours per week and total years of juggling practice) could mainly account for the absence of structural changes in cortical networks in response to motor training. Indeed, experts were selected among a population of jugglers able to juggle at five balls for at least for $20 \mathrm{~s}$. With a weekly practice of $4.3 \mathrm{~h}$ (range: $1-10 \mathrm{~h} / \mathrm{week}$ ), they practiced juggling for 11 years (range: 3-19 years with 2 jugglers who did not disclose how many years they had practiced). These results also highlight that structural changes at a local level do not presume structural changes at an inter-regional level.

When focusing on investigations comparing resting-state functional connectivity between motor experts and non-expert subjects (Di et al. 2012; Fauvel et al. 2014; Li et al. 2015; Luo et al. 2012, 2014; Tan et al. 2017), one can see that all the research teams used a seed-based approach. This approach makes it possible to detect a temporal correlation between a predefined area (i.e., the seed) and all the other brain areas. Seed areas are chosen by taking into account the characteristics of the population and/or the processes being examined. In the present reviewed studies, seeds were selected through four different methods. First, seeds were defined on the basis of previous research works (Luo et al. 2012). Second, they were identified based on functional differences between experts and non-experts: (i) in local functional connectivity (Luo et al. 2014) and (ii) in both short-range and long-range functional connectivity density ( $\mathrm{Li}$ et al. 2015). Third, seeds were chosen on the basis of structural differences in gray matter volume between experts and non-experts (Fauvel et al. 2014; Tan et al. 2017). Lastly, seeds were determined on the basis of structural and functional alterations i.e. greater gray matter concentration associated with higher/lower amplitudes of low-frequency fluctuations (ALFFs) (Di et al. 2012). Except for studies by Di et al. (2012) and Luo et al. (2012), 
clusters where structural and functional cortical markers were significantly higher in motor experts than in non-experts were used for subsequent functional connectivity analyses.

When functional connectivity data are analyzed, we observe a connectivity pattern heterogeneity in terms of direction and locus of changes. More specifically, when comparing experts to non-experts, two different patterns of practice-related changes in distinct networks have been detected: an inter-system connection increment in skilled samples (Fauvel et al. 2014; Li et al. 2015; Luo et al. 2012, 2014; Tan et al. 2017) and a combination of increased and decreased inter-system connections in motor experts (Di et al. 2012).

Five out of six studies revealed an inter-system connection increment in skilled samples. Luo et al. (2012) showed that functional connectivity was greater in the motor, visual, auditory and somatosensory cortices of musicians compared to that in musically naive subjects. To broaden their results, Luo et al. (2012) also conducted an effective connectivity analysis assessed via a Granger causal analysis (Goebel et al. 2003; Roebroeck et al. 2005). Luo et al. (2012) found different effective connectivity patterns in musicians and musically naive subjects and a greater outflow-inflow degree within the left primary auditory cortex in musicians. More specifically, the outflow degree in musicians was from the primary auditory cortex and VI area to others nodes whereas it was from primary somatosensory cortex and VII area in musically naive subjects. The inflow degree in musicians was at the primary motor cortex and VII area from other nodes whereas it was at the primary auditory cortex and VI area in their non-expert counterparts. These results imply that the auditory cortex is responsible for triggering these functional connection changes induced by an accumulated substantial musical experience. In 2014, using the insula, temporo-parietal junction, and anterior cingulated cortex as seeds, Luo et al. revealed, after an extensive and intensive musical training, increases in distant functional connectivity in areas, of which most are recognized to belong to the salience network. The salience network is recognized to be involved in switching between the default-mode and task-related brain networks (Menon and Uddin 2010; Seeley et al. 2007). When a salient stimulus is perceived, the salience network is at play. It allows task-related information to be processed by enrolling brain areas mediating attention, working memory and higher order cognitive processes while deactivating the default-mode network in order to keep attention focused on task-relevant stimuli and goals. In 2015, using the putamen, primary motor and somatosensory cortices, and right superior occipital gyri as seeds, the same research group showed, in modern dancers, increased functional connectivity between the putamen and right middle cingulate gyrus and between the primary motor and somatosensory cortices ( $\mathrm{Li}$ et al. 2015). Interestingly, the putamen is recognized to be engaged in motor control processes (Brown et al. 2006) whereas the middle cingulate gyrus is recognized to be involved in attentional and executive processing (Van Veel et al., 2001), and in decision making (Kennerley et al., 2006). In short, this study reveals a functional connectivity increment within sensorimotor-related brain networks in proficient dancers which can be interpreted as a greater sensorimotor control and integration. Interestingly, no different functional connectivity was observed with the right superior occipital seed between dancers and non-dancers ( $\mathrm{Li}$ et al. 2015). Using the right cingulate gyrus, left superior temporal gyrus, and right inferior orbitofrontal gyrus as seeds, Fauvel et al. (2014) also revealed that these areas exhibited greater resting-state functional connectivity with highlevel cognitive areas, primary sensorimotor cortex, and subcortical structures in musicians than in musically naive subjects. Interestingly, enhanced functional connectivity at rest was not found among musically naive subjects. Very recently, using the left anterior insula, inferior frontal gyrus, inferior parietal lobule and the right anterior cingulate cortex, precuneus as seed areas, Tan et al. (2017) showed that these areas displayed higher functional connectivity with areas belonging to the default mode, salience and executive control networks in first-class basket-ball players compared to non-athletes.
The remaining study by Di et al. (2012) showed a combination of increased and decreased inter-system connections in expert badminton players relative to non-experts. Using medial cerebellum and left superior parietal lobule as seed areas where respectively a higher ALFF and a weaker ALFF have been observed in badminton players, Di et al. (2012), on one hand, revealed a functional connectivity decrease between the medial cerebellum and anterior cingulate cortex in these skilled athletes. On the other hand, they registered functional connectivity increase and decrease between the superior parietal lobule and different parts of the middle frontal cortex (BA6 for increase and BA9 for decrease). Interestingly, Di et al. (2012) also found no functional changes in cortical networks in response to motor training when anterior and posterior cerebella were used as seeds and displayed higher gray matter concentration in expert badminton players compared to controls.

Regarding the seed-based functional connectivity (FC) results, the reader may wonder about

the impact of local alterations, be they structural or functional, on functional connectivity.

In view of the results from Di et al. (2012), Fauvel et al. (2014) and Tan et al. (2017), greater structural changes observed at a local level in motor experts do not presume functional changes at an inter-regional level as revealed by the two kinds of patterns: (i) greater gray matter alteration within a particular seed area associated with no alteration in functional connectivity ("increase-absence of change" pattern; Di et al. 2012), and (ii) greater gray matter alteration within a particular seed area coexisting with increase in functional connectivity ("increase-increase" pattern, Fauvel et al. 2014; Tan et al. 2017). In other words, present results are inconclusive to infer function from structure. To shed more light on the impact of structural alterations on functional connectivity or more generally on the structure-function relationship, comparing structural connectivity with resting-state functional connectivity or comparing structural network topology with functional network topology warrants further consideration. However, this tight relationship is far from being simple. For instance, though there tends to be a one-to-one correspondence between structural and functional connectivity (i.e. a high degree of similarity between structural and functional connectivity maps) (e.g., Hagmann et al. 2008; Hermundstad et al. 2013; Koch et al. 2002; Skudlarski et al. 2008), some studies showed discrepant findings. Greicius et al. (2003) and Honey et al. (2009) observed the existence of functional connectivity between two given brain areas which do not share a (direct) structural connection. More information related to this topic can be found in Wang et al. review (2015).

In view of the results from Di et al. (2012), Li et al. (2015), and Luo et al. (2014), greater functional changes observed at a local level in motor experts do not presume functional changes at an inter-regional level as revealed by three kinds of patterns: (i) greater functional alteration within a particular seed area associated with no alteration in functional connectivity ("increase-absence of change" pattern; Li et al. 2015), (ii) greater functional alteration within a particular seed area associated with increase in functional connectivity ("increase-increase" pattern; Li et al. 2015; Luo et al. 2014), (iii) greater functional alteration within a particular seed area associated with decrease in functional connectivity ("increase-decrease" pattern; Di et al., 2012). Along the same lines, weaker functional changes observed at a local level in motor experts do not presume functional changes at an inter-regional level as revealed by two kinds of patterns: (i) weaker functional alteration within a particular seed area associated with increase in functional connectivity ("decrease-increase" pattern; Di et al. 2012), and (ii) weaker functional alteration within a particular seed area associated with decrease in functional connectivity ("decrease-decrease" pattern; Di et al. 2012). At this point, it is relevant for the reader to keep in mind that activation across a large array of brain areas does not allow disclosure of information related to interactions between areas. Furthermore, it is not because an interaction between a particular area with 
other areas is altered over the course of motor training that the level of activation within this particular area changes. It may remain stable (Kelly and Garavan, 2005). In the present review, this case cannot be appraised as seeds were selected on the basis of cortical differences between experts and non-experts.

To conclude, when functional interactions between pairs of brain components are analyzed, we observe a connectivity pattern heterogeneity in terms of locus of changes and direction. The heterogeneity in terms of locus of changes could be explained by the selection of seed areas which differs a great deal across studies and by the fact that a structural or functional local alteration within a particular area does necessarily lead to functional connectivity changes between that area and other areas. Additionally, the characteristic diversity of the practiced motor tasks: music (i.e., violin, cello, guitar, flute, recorder, trumpet, clarinet, piano, accordion) vs sport (i.e., badminton, modern dance) could account for these discrepancies (see Box 2, see Bangert and Schlaug, 2006)

As for the connectivity pattern heterogeneity in terms of direction of changes, it could be interpreted by a consolidation of the essential connections dedicated to the task at hand and a pruning of the superfluous ones. This suggestion could be sort of as an example of the expansion-renormalization process that reflects the cortical plasticity mechanisms, as supported by Wenger et al. (2017a,b). Of course, this is pure speculation and warrants further investigation.

But, it remains no less true that interpreting the coexistence of increased and decreased connectivity between various brain areas questions our understanding of the mechanisms underlying cortical changes induced by a lengthy-extensive motor training. Hence, comparing different studies is complex. Using a network approach based on graph theory seems to us necessary as such an approach strengthens the perspective of connectivity in a large-scale complex brain networks (McIntosh and Korostil 2008) and presents concepts, such as clustering, path length, modularity, which can facilitate the identification of organizational patterns in brain networks and the comparisons across studies.

\subsection{Investigating structural and functional changes in cortical networks: $a$ graph theoryapproach}

In the present review, 3 out of 23 studies investigate structural and functional changes by examining connectivity in a large-scale complex brain networks, reflected by a higher order of interaction by means of graph theory analysis (Li et al. 2014; Wang et al. 2013, 2016).

Using graph theory, the seminal works by Wang et al. (2013, 2016) compared the topological properties of cortical structural/anatomical and functional networks between (Chinese) world class gymnasts and sedentary subjects. They found that the structural and functional networks of outstanding experts and controls displayed both small-world properties. Small-world networks, defined by high clustering and short path length, are considered as systems that are both globally and locally efficient (Latora and Marchiori 2001). Efficiency in a network is an indicator of how well information is conveyed within the cerebral cortex. High global efficiency is interpreted as an integrated network able to ensure rapid information transmission between and across remote cortical areas. On the other hand, high local efficiency indicates that the network is highly fault-tolerant which means that it efficiently transfers the information within the neighbors of a particular node when this node is removed (Latora and Marchiori 2001).

This research group also detected higher global and local efficiency in structural networks of motor expert performers compared to those of non-experts, whereas the reverse pattern was found in functional networks. These findings were associated to shorter characteristics path lengths in experts and to no significant clustering differences between experts and non-experts in structural networks, and to longer characteristics path lengths and lower clustering in experts in functional networks. Altogether, these results provide evidence that after extensive and intensive motor training, human cortical networks have been transformed. However, directions of changes in structural network topology do not match those observed in functional network topology. In addition, when compared to non-experts, Wang et al. (2016) found, for experts, intra-and inter-modular functional reorganizations, as reflected by functional decreases in the cerebellum, cingulo-opercular and fronto-parietal modules. This could be explained by a greater efficiency in the expert brains induced by lengthy-extensive motor training.

Using graph theory, Li et al. (2014) compared the topological properties of white matter structural networks between female musicians, playing the piano, the Chinese zither, and the accordion, and non-musicians. Though the structural networks of experts and non-experts displayed both small-world properties, no significant differences in network efficiency, whether it be global or local, were found between these two kinds of populations. However, on a regional level, compared to non-musicians, higher white matter connectivity strength was revealed within the SMA, primary visual cortex and caudate nucleus in musicians as well as greater weighted clustering coefficient within the olfactory cortex, medial superior frontal gyrus, gyrus rectus, lingual gyrus, supramarginal gyrus and pallidum. In musicians, Li et al. (2014) also depicted the coexistence of two cortical phenomena: (i) higher nodal betweenness centrality within sensorimotor areas (i.e., precentral gyrus, SMA) and (ii) weaker nodal betweenness centrality within visual areas (i.e., middle and inferior occipital gyrus) and within the caudate nucleus, an area recognized to be involved in emotional processing (Carretie et al. 2009). Taken together, these findings suggest that musicians have improved information transmission efficiencies in local white matter networks between areas known to be involved in sensory, motor and emotional processing.

\section{Box 3}

Basic Network Terminology used in Wang et al. (2013) study (Achard and Bullmore, 2007; Latora and Marchiori 2001; Stam and van Straaten 2012).

Graph: Mathematical representation of a network.

Vertex: Node of the network. A node can represent a particular brain region

Edge: A connection or a relation between two vertices/nodes

Module: A subset of highly interconnected nodes that are re-

latively sparsely connected to nodes in other modules

Global parameters

Characteristic path length: Number of edges in the shortest path between two vertices/nodes

Clustering coefficient: Number of edges present between the neighbors of a vertex/node divided by the total possible number of edges between the neighbors

Small-world network: A network that combined high clustering with short path lengths

Global efficiency: Inverse of the characteristics path length

Local efficiency: Efficiency computed from the set of neighbors of a vertex/node

Regional parameters

Degree: the degree of a vertex/node is the number of edges connected to this node

Regional efficiency for a given vertex/node: Inverse of mean harmonic shortest path length between a given vertex/node and all the other vertexes/nodes of the network (Achard and Bullmore, 2007)

Betweenness centrality: Fraction of all shortest paths that pass through a particular vertex or edge

\subsection{Using task-free fMRI}

The increasingly widespread use of task-free fMRI (i.e., use of resting-states) also begs the question of whether brain architecture 
when at rest closely mirrors architecture at play when performing tasks such as cognitive or motor tasks. Opinions differ in the literature. Some agree with the existence of a close relationship between the topography of task-free and task-evoked functional networks (Lewis et al. 2009; Smith et al. 2009) whereas others are of mixed opinion (Buckner et al. 2013; Mennes et al. 2013). For instance, Mennes et al. (2013) revealed a tight relationship between these two architectures within the default mode, and task-positive areas (dorsal attentional areas, dorso-lateral and ventral prefrontal areas, insula, and the supplementary motor area) but not within the subcortical, limbic, and primary sensory motor areas. Consequently, they came to the conclusion that a resting-state functional connectivity approach offers a partial comprehension of the functional architecture of the brain. Recently, Cole et al. (2014) uncovered the existence of an intrinsic standard architecture of functional brain organization that strongly contributes to mapping the restingstate network architecture and to a lesser extent the task-evoked network architecture. More specifically, the network architecture during a specific task is principally shaped by the intrinsic standard architecture of functional brain organization and additionally by a limited set of task-general and task-specific evoked changes confined to a weak number of connections. This finding will help bridge the gap between task-free and task-evoked functional connectivity findings in the literature and offers a better understanding of the functional architecture of the brain. Hence, using task-free fMRI appears to us justified in order to deepen our knowledge of mechanisms that underpin motor expertise.

\section{Conclusion}

This review paper provides insights into the neural mechanisms underlying motor expertise which is defined as being the result of intensive and extensive motor training reflected by a sheer volume of at least several thousand hours. It shows that $56.5 \%$ of the studies investigated changes on a local level, $30.5 \%$ on a network level where interactions between pairs of brain components were considered, and the remaining $13 \%$ on a "higher order" of interaction known as network organization and based on graph theory.

Taken together, this review's findings support that structural and functional changes occur in response to lengthy-extensive motor training and that this training shapes the human brain in areas related to the execution of the task, as recognized by the scientific community (Buschkuehl et al. 2012; Doyon et al. 2002; Guida et al. 2012; Jueptner et al. 1997). Consequently, observing practice-related changes in different areas/systems among experts from all walks of life was to be expected. However, it should be highlighted that changes have also been found in common areas/systems in musicians and athletes. Common intrinsic characteristics shared by seemingly dissimilar disciplines count account for this.

This review also points out the coexistence of different cortical patterns within and between various brain areas/systems. First, regardless of the cortical indices, be they structural or functional, three patterns of practice-related changes have been found: increase, decrease, and a combination of increase and decrease. Increase means that higher values of a specific cortical measure are observed in experts compared to non-experts while decrease describes the reverse pattern. Combination of increase and decrease reflects the coexistence of different patterns in skilled samples i.e. greater and weaker cortical measures in experts when compared to non-experts. One possible mechanism to account for these observed divergent patterns could be that brain areas/systems evolve over time at different space and time scale. Taking this idea further and as supported by Wenger et al. (2017a,b), different areas may follow the same change patterns but at different rates by going through different stages which are expansion, selection and renormalization of brain activity and structure. Of course, this is speculation. Going further, one can wonder whether the expansion-renormalization model may account for the diverse manifestations of cortical plasticity. Second, along the same lines, the impact of local changes on inter-regional changes still remains unclear. In this review, it has been seen that greater structural changes observed at a local level in motor experts do not presume changes on structural and functional connectivity (Gerber et al. 2014 for structural connectivity; Di et al. 2012, Fauvel et al. 2014, Tan et al. 2017 for functional connectivity) and that greater or lower functional changes observed at a local level in experts do not infer changes on functional connectivity (Di et al. 2012, Li et al. 2015, Luo et al. 2014). This has been reflected by the existence of five patterns for "local changes-inter-regional changes": "increaseabsence of change", "increase-increase", "increase-decrease", "decrease-increase", and "decrease-decrease" patterns.

Consequently, such coexisting different patterns at a given point in time makes it difficult to interpret these data and consequently challenges our understanding of the mechanisms underlying cortical changes induced by a lengthy-extensive motor training. A more elaborate approach, such as a large-scale complex network approach based on graph theory seems to us necessary as it can facilitate the identification of organizational patterns in brain network and the comparisons across studies.

\section{Future perspectives}

In light of the results of the present review, there are still mechanisms which are not yet fully understood such as the coexistence of various patterns of practice-related changes, the impact of local changes on inter-regional changes, or the structure-function relationship. Future research should pay careful attention to any factors that might have influenced findings in previous research. Some recommendations are provided.

First, diversity of sample constitution could be a possible cause for inconsistencies observed throughout this review. To name but a few: (i) diversity of participant gender across studies (i.e., male, female or both genders), (ii) diversity of experts' selection within a study (i.e., different types of instruments played in musical studies, different types of sport disciplines practiced by experts), (iii) diversity of experts' experience within a study (i.e., different initial onset of training, different weekly training hours), (iv) lack of match between control subjects and expert cohorts (i.e., possession by controls of motor experience in a different or a similar discipline than that of experts). Consequently, aware that recruiting motor expert performers and matched controls is not an easy task, careful attention has to be paid to this selection.

Second, aware that studies of a cross-sectional nature do not enable us to understand how particular attributes acquired by motor expert performers have developed over time, following the same cohort of individuals over long time periods, i.e. several years, would seems to be an effective approach. However, in concrete terms and as rightly pointed out by Wang et al. (2013), this is hardly feasible, logistically difficult and time-consuming as evidenced by the very few studies which did it: Hyde et al. (2009) with 6-year old children following a 15month musical training and Woollett and Maguire (2011) with adults undertaking a 3-4 year-training program to become licensed taxi drivers. Consequently, combining cross-sectional and longitudinal designs, i.e. following several differently aged cohorts possessing different levels of motor expertise over time, e.g. 2 or 3 years, could be an acceptable compromise. This could allow us to distinguish the effects of genetics or nature (e.g., innate giftedness, hereditary predispositions) from environmental influences or nurture (e.g., motor training, socioeconomic status, supportive social environment) since the same cohorts would be studied over time.

Third, some neuroimaging techniques which have been used in the reviewed studies rely on derived measures that do not provide access to the plasticity process. Therefore, it is fundamental to develop histological, biochemical studies and MRI in animals to deepen understanding of micro-level changes underlying structural changes that neuroimaging techniques cannot reveal (e.g., Keifer et al. 2015; Lerch et al. 2011). 
Lastly, associating various imaging modalities (e.g., sMRI, DTI, taskfree and task-related fMRIs) would allow researchers: (i) to lower the limitations of using a single modality, (ii) to obtain a more refined picture of brain architecture (Wang et al. 2014), and (iii) to clarify the structure-function relationship. Besides, as suggested by Steele et al. (2012), associating these techniques with behavioral performance measures can display how cortical changes are associated with behavior. Using new analysis methods, such as multi-voxel pattern analysis (MVPA; Wiestler and Diedrichsen, 2013) or recent developed algorithms to detect time evolving communities (Bassett et al., 2013) could also be of help to shed new light on the mechanisms underlying cortical changes resulting from an extensive and intensive motor training. At least, conducting analyses at a network level with measures based on graph theory seem to be promising to understand the mechanisms underpinning motor expertise, and to improve our comprehension of brain architecture.

\section{Declaration of Competing Interest}

This review was conducted in the absence of any commercial or financial relationship that could be construed as a potential conflict of interest.

\section{Acknowledgement} looked.

Apologies to any authors whose research was mistakenly over-

\section{Appendix A. Supplementary data}

Supplementary data to this article can be found online at https:// doi.org/10.1016/j.brainres.2019.146323.

\section{References}

Achard, S., Bullmore, E., 2007. Efficiency and cost of economical brain functional networks. PLoS Comput. Biol. 3, e17. https://doi.org/10.1371/journal.pcbi.0030017.

Alexander, A.L., Lee, J.E., Lazar, M., Field, S., 2007. Diffusion tensor imaging of the brain. Neurotherapeutics 4, 316-329. https://doi.org/10.1016/j.nurt.2007.05.011.

Assaf, Y., Pasternak, O., 2008. Diffusion tensor imaging (DTI)-based white matter mapping in brain research: a review. J. Mol. Neurosci. 34, 51-61. https://doi.org/10. 1007/s12031-007-0029-0.

Bangert, M., Schlaug, G., 2006. Specialization of the specialized in features of external human brain morphology. Eur. J. Neurosci. 24, 1832-1834. https://doi.org/10. 1111/j.1460-9568.2006.05031.x.

Barabasi, A.L., Albert, R., 1999. Emergence of scaling in random networks. Science 286 509-512. https://doi.org/10.1126/science.286.5439.50.

Bassett, D.S., Wymbs, N.F., Rombach, M.P., Porter, M.A., Mucha, P.J., Grafton, S.T., 2013. Task-based core-periphery organization of human brain dynamics. PLoS Comput. Biol. 9, e1003171. https://doi.org/10.1371/journal.pcbi.1003171.

Beaulieu, C., 2002. The basic of anisotropic water diffusion in the nervous system - a technical review. NMR Biomed. 15, 435-455.

Beaulieu, C., 2009. The biological basis of anisotropic water diffusion. In: Johanson-Berg, H., Behrens, T.E.J. (Eds.), Diffusion MRI: From Quantitative Measurement to In-vivo. Neuroanatomy. Elsevier, London, pp. 105-126.

Bengtsson, S.L., Nagy, Z., Skare, S., Forsman, L., Forssberg, H., Ullen, F., 2005. Extensive piano practicing has regionally specific effects on white matter development. Nat. Neurosci. 8, 1148-1150. https://doi.org/10.1038/nn1516.

Bermudez, P., Lerch, J.P., Evans, A.C., Zatorre, R.J., 2009. Neuroanatomical correlates of musicianship as revealed by cortical thickness and voxel-based morphometry. Cereb. Cortex 19, 1583-1596. https://doi.org/10.1093/cerco/bhn196.

Bezzola, L., Merillat, S., Jancke, L., 2012. Motor training-induced neuroplasticity. J. Gerontopsychol. Geriatric Psychiatry 25, 189-197. https://doi.org/10.1024/16629647/a000070.

Brown, S., Martinez, M.J., Parsons, L.M., 2006. The neural basis of human dance. Cereb. Cortex 16, 1157-1167. https://doi.org/10.1093/cercor/bhj057.

Buchel, C., Friston, K.J., 1997. Modulation of connectivity in visual pathways by attention: cortical interactions evaluated with structural equation modelling and fMRI. Cereb. Cortex 7, 768-778.

Buckner, R.L., Krienen,F.M., Yeo, B.T.T., 2013. Opportunitiesandlimitationsofintrinsic functional connectivity MRI. Nat. Neurosci. 16, 832-837. https://doi.org/10.1038/ nn. 3423 .

Bullmore, E., Sporns, O., 2009. Complex brain networks: graph theoretical analysis of structural and functional systems. Nat. Rev. Neurosci. 10, 186-198 10.1038:nrn2575.

Buschkuehl, M., Jaeggi, S.M., Jonides, J., 2012. Neuronal effects following working memory training. Dev. Cogn. Neurosci. 15, 167-179. https://doi.org/10.1016/j.DCN. 2011.10.001.

Calvo-Merino, B., Glaser, D.E., Grezes, J., Passingham, R.E., Haggard, P., 2005. Action observation and acquired motor skills: an fMRI study with expert dancers. Cereb. Cortex 15, 1243-1249. https://doi.org/10.1093/cercor/bhi007.

Carretie, L., Rios, M., de la Gandara, B.S., Tapia, M., Albert, J., Lopez-Martin, S., AlvarezLinera, J., 2009. The striatum beyond reward: caudate responds intensely to unpleasant pictures. Neuroscience 164, 1615-1622. https://doi.org/10.1016/j. neuroscience.2009.09.031.

Chang, Y., 2014. Reorganization and plastic changes of the human brain associated with skill learning and expertise. Front. Hum. Neurosci. 8, 35. https://doi.org/10.3389/ fnhum.2014.00035.

Cole, M.W., Bassett, D.S., Power, J.D., Braver, T.S., Petersen, S.E., 2014. Intrinsic and task-evoked network architectures of the human brain. Neuron 83, 238-251. https:// doi.org/10.1016/j.neuron.2014.05.014.

Concha,L., 2014. A macroscopic view of microstructure: Using diffusion-weighted images to infer damage, repair, and plasticity of white matter. Neuroscience 276, 14-28. https://doi.org/10.1016/j.neuroscience.2013.09.004.

Concha, L., Livy, D.J., Beaulieu, C., Wheatley, B.M., Gross, D.W., 2010. In vivo diffusion tensor imaging and histopathology of the fimbria-fornix in temporal lobe epilepsy. J. Neurosci. 30, 996-1002. https://doi.org/10.1523/jneurosci.1619-09.2010.

Debarnot, U., Sperduti, M., Di Rienzo, F., Guillot, A., 2014. Expertsbodies, expertsminds: how physical and mental training shape the brain. Front. Hum. Neurosci. 8, 280. https://doi.org/10.3389/fnhum.2014.00280.

Di, X., Zhu, S., Jin, H., Wang, P., Ye, Z., Zhou, K., Zhuo, Y., Rao, H., 2012. Altered resting brain function and structure in professional badminton players. Brain Connect. 2, 225-233. https://doi.org/10.1089/brain.2011.0050.

Douw, L., Schoonheim, M.M., Landi, D., van der Meer, Geurts JJG, Reijneveld, J.C., Klei, M., Stam, C.J., 2011. Cognition is related to resting-state small-world network topology: an magnetoencephalographic study. Neuroscience 175, 169-177. https://doi org/10.1016/j.neuroscience.2010.11.039.

Doyon, J., Song, A.W., Karni, A., Lalonde, F., Adams, M.M., Ungerleider, L.G., 2002. Experience-dependent changes in cerebellar contributions to motor sequence learning. Proc. Natl. Acad. Sci. 99 (2), 1017-1022. https://doi.org/10.1073/pnas. 022615199.

Ericsson, K.A., Lehmann, A.C., 1996. Expert and exceptional performance: evidence of maximal adaptation to task constraints. Annu. Rev. Psychol. 47, 273-305. https:// doi.org/10.1146/annurev.psych. 47.1.273.

Fauvel, B., Groussard, M., Chételat, G., Fouquet, M., Landeau, B., Eustache, F., Desgranges, B., Platel, H., 2014. Morphological brain plasticity induced by musical expertise is accompanied by modulation of functional connectivity at rest. Neuroimage 90, 179-188. https://doi.org/10.1016/j.neuroimage.2013.12.065.

Filippi, M., Ceccarelli, A., Pagani, E., Gatti, R., Rossi, A., Stefanelli, L., Falini, A., Comi, G., Rocca, M.A., 2010. Motor learning in healthy humans is associated to gray matter changes: a tensor-based morphometry study. PLoS One 5, e10198. https://doi.org/ 10.1371/journal.pone.0010198.

Freeman, J., Brouwer, G.J., Heeger, D.J., Merriam, E.P., 2011. Orientation decoding depends on maps, not columns. J. Neurosci. 31, 4792-4804. https://doi.org/10 1523/jneurosci.5160-10.2011.

Friston, K.J., 1994. Functional and effective connectivity in neuroimaging: a synthesis Hum. Brain Mapp. 2, 56-78. https://doi.org/10.1002/hbm.460020107.

Friston, K.J., 2011. Functional and effective connectivity: a review. Brain Connect. 1 , 13-36. https://doi.org/10.1089/brain.2011.0008.

Friston, K.J., Harrison, L., Penny, W., 2003. Dynamic causal modelling. Neuroimage 19, 1273-1302. https://doi.org/10.1016/S1053-8119(03)00202-7.

Fu, M., Zuo, Y., 2011. Experience-dependent structural plasticity in the cortex. Trends Neurosci. 34, 177-187. https://doi.org/10.1016/j.tins.2011.02.001.

Gaser, C., Schlaug, G., 2003. Brain structures differ between musicians and non-musicians. J. Neurosci. 23, 9240-9245.

Gerber, P., Schlaffke, L., Heba, S., Greenlee, M.W., Schultz, T., Schmidt-Wilcke, T., 2014 Juggling revisited - a voxel-based morphometry study with expert jugglers. Neuroimage 95, 320-325. https://doi.org/10.1016/j.neuroimage.2014.04.023.

Goebel, R., Roebroeck, A., Kim, D.S.,Formisano, E., 2003. Investigating directed cortical interactions in time-resolved fMRI data using vector autoregressive modeling and Granger causality mapping. Magn. Reson. Imaging 21, 1251-1261. https://doi.org/ 10.1016/j.mri.2003.08.026.

Goldberg, G., 1985. Supplementary motor area structure and function: review and hypotheses. Behav. Brain Sci. 8, 567-588. https://doi.org/10.1017/ S0140525X00045167

Good, C.D., Johnsrude, I., Ashburner, J., Henson, R.N., Friston, K.J., Frackowiak, R.S. 2001. Cerebral asymmetry and the effects of sex and handedness on brain structure: a voxel-based morphometric analysis of 465 normal adult human brains. Neuroimage 14, 685-700. https://doi.org/10.1006/nimg.2001.0857.

Gootjes, L., Bouma, A., van Strien, J.W., Scheltens, P., Stam, C.J., 2006. Attention modulates hemispheric differences in functional connectivity evidence from MEG recordings. Neuroimage 30, 245-253. https://doi.org/10.1016/j.neuroimage.2005.09. 015.

Gowen, E., Miall, R., 2007. Differentiation between external and internal cuing: an fMRI study comparing tracing with drawing. Neuroimage 36, 396-410. https://doi.org/10. 1016/j.neuroimage.2007.03.005.

Grainger, J., Lété, B., Bertand, D., Dufau, S., Ziegler, J.C., 2012. Evidence for multiple routes in learning to read. Cognition 123, 280-292. https://doi.org/10.1016/j. cognition.2012.01.003

Greicius, M.D., Krasnow, B., Reiss, A.L., Menon, V., 2003. Functional connectivity in the resting brain: a network analysis of the default mode hypothesis. PNAS USA 100 , 253-258. https://doi.org/10.1073/pnas.0135058100. 
Guida, A., Gobet, F., Tardieu, H., Nicolas, S., 2012. How chunks, long-term working memory and templates offer a cognitive explanation for neuroimaging data on expertise acquision: a two-stage framework. Brain Cogn. 79, 221-244. https://doi.org/ 10.1016/j.bandc.2012.01.010.

Hagmann, P., Cammoun, L., Gigandet, X., Meuli, R., Honey, C.J., Wedeen, V.J., Sporns, O., 2008. Mapping the structural core of human cerebral cortex. PLoS Biol. 6, e159. https://doi.org/10.1371/journal.pbio.0060159.

Hagmann, P., Jonasson, L., Maeder, P., Thiran, J.P., van Wedeen, J., Meuli, R., 2006. Understanding diffusion MR imaging techniques: from scalar diffusion-weighted imaging to diffusion tensor imaging and beyond. Radiographics 26, S205-S223. https://doi.org/10.1148/rg.26si065510.

Han, Y., Yang, H., Lv, Y.T., Zhu, C.Z., He, Y., Tang, H.H., Gong, Q.Y., Luo, Y.J., Zang, Y.F., Dong, Q., 2009. Gray matter density and white matter integrity in pianists' brain: a combined structural and diffusion tensor MRI study. Neurosci. Lett. 459, 3-6. https:// doi.org/10.1016/j.neulet.2008.07.056.

Hanggi, J., Koeneke, S., Bezzola, L., Jancke, L., 2010. Structural neuroplasticity in the sensorimotor network of professional female ballet dancers. Hum. Brain Mapp. 31 , 1196-1206. https://doi.org/10.1002/hbm.20928.

Hardwick, R.M., Rottschy, C., Miall, R.C., Eickhoff, S.B., 2013. A quantitative metaanalysis and review of motor learning in the human brain. Neuroimage 67, 283-297. https://doi.org/10.1016/j.neuroimage.2012.11.020.

Haslinger, B., Erhard, P., Altenmuller, E., Hennenlotter, A., Schwaiger, M., Grafin von Einsiedel, H., Rummeny, E., Conrad, B., Ceballos-Baumann, A.O., 2004. Reduced recruitment of motor association areas during bimanual coordination in concert pianists. Hum. Brain Mapp. 22, 206-215. https://doi.org/10.1002/hbm.20028.

He, Y., Wang, J., Wang, L., Chen, Z.J., Yan, C., Yang, H., Tang, H., Zhu, C., Gong, Q., Zang, Y., Evans, A.C., 2009. Uncovering intrinsic modular organization of spontaneous brain activity in humans. PLoS One 4, e5226. https://doi.org/10.1371/journal. pone.0005226

Herholz, S.C., Zatorre, R.J., 2012. Musical training as a framework for brain plasticity: behaviour, function, and structure. Neuron 76, 486-502. https://doi.org/10.1016/j. neuron.2012.10.011.

Hermundstad, A.M., Bassett, D.S., Brown, K.S., Aminoff, E.M., Clewett, D., Freeman, S., Frithsen, A., Johnson, A., Tipper, C.M., Miller, M.B., Grafton, S.T., Carlson, J.M., 2013. Structural foundations of resting-state and task-based functional connectivity in the human brain. PNAS USA 110, 6169-6174. https://doi.org/10.1073/pnas. 1219562110 .

Imfeld, A., Oechslin, M.S., Meyer, M.,Loenneker, T., Jancke, L., 2009. White matter plasticity in the corticospinal tract of musicians: a diffusion tensor imaging study. Neuroimage 46, 600-607. https://doi.org/10.1016/j.neuroimage.2009.02.025.

Honey, C., Sporns, O., Cammoun, L., Gigandet, X., Thiran, J.P., Meuli, R., Hagmann, P., 2009. Predicting human resting-state functional connectivity from structural connectivity. PNAS USA 106, 2035-2040. https://doi.org/10.1073/pnas.0811168106.

Huang, R., Lu, M., Song, Z., Wang, J., 2015. Long-term intensive training induced brain structural changes in world class gymnasts. Brain Struct. Funct. 220, 625-644. https://doi.org/10.1007/s00429-013-0677-5.

Hufner, K., Binetti, C., Hamilton, D.A., Stephan, T., Flanagin, V.L., Linn, J., Labudda, K., Markowitsch, H., Jahn, K., Strupp, M., Brandt, T., 2011. Structural and functional plasticity of the hippocampal formation in professional dancers and slackliners. Hippocampus 21, 855-865. https://doi.org/10.1002/hipo.20801.

Hutton, C., de Vitae, E., Ashburner, J., Deichmann, R., Turner, R., 2008. Voxel-based cortical thickness measurements in MRI. Neuroimage 40, 1701-1710. https://doi org/10.1016/j.neuroimage.2008.01.027.

Hyde, K.L., Lerch, J., Norton, A., Forgeard, M., Winner, E., Evans, A.C., Schlaug, G., 2009. Musical training shapes structural brain development. J. Neurosci. 29, 3019-3025. https://doi.org/10.1523/JNEUROSCI.5118-08.2009.

James, C.E., Oechslin, M.S., van de Ville, D., Hauert, C.A., Descloux, C., Lazeyras, F., 2014. Musical training intensity yields opposite effects on grey matter density in cognitive versus sensorimotor networks. Brain Struct. Funct. 219, 353-366. https:// doi.org/10.1007/s00429-013-0504-z.

Jancke, L., 2009. Music drives brain plasticity F1000. Biol. Rep. 1, 78. https://doi.org/10. 3410/B1-78.

Jancke, L., Koeneke, S., Hoppe, A., Rominger, C., Hanggi, J., 2009. The architecture of the golfer's brain. PLoS One 4, e4785. https://doi.org/10.1371/journal.pone.0004785.

Jensen, J.L., Marstrand, P.C., Nielsen, J.B., 2005. Motor skill training and strength training are associated with different plastic changes in the central nervous system. J. Appl. Physiol. 99, 1558-1568. https://doi.org/10.1152/japplphysiol.01408.2004.

Jeurissen, B., Leemans, A., Tournier, J.D., Jones, D.K., Sijbers, J., 2013. Investigating the prevalence of complex fiber configurations in white matter tissue with diffusion magnetic resonance imaging. Hum. Brain Mapp. 34, 2747-2766. https://doi.org/10. 1002/hbm.22099.

Jueptner, M., Stephan, K.M.,Frith, C.D., Brooks, D.J., Frackowiak, R.S.,Passingham, R.E., 1997. Anatomy of motor learning. I. Frontal cortex and attention to action. J. Neurophysiol. 77, 1313-1324. https://doi.org/10.1152/jn.1997.77.3.1313.

Keifer, O.P., Hurt, R.C., Gutman, D.A., Keilholz, S.D., Gourley, S.L., Ressler, K.J., 2015. Voxel-based morphometry predicts shifts in dendritic spine density and morphology with auditory fear conditioning. Nat. Commun. 6, 7582. https://doi.org/10.1038/ ncomms 8582 .

Keller, P.E., 2001. Attentional resource allocation in musical ensemble performance. Psychology of music. Psychol. Music 29, 20-38.

Kelly, A.M.C., Garavan, H., 2005. Human functional neuroimaging of brain changes associated with practice. Cereb. Cortex 15, 1089-1102. https://doi.org/10.1093/ cercor/bhiO05

Kennerley, S.W., Walton, M.E., Behrens, T.E., Buckley, M.J., Rushworth, M.F., 2006. Optimal decision making and the anterior cingulate cortex. Nat. Neurosci. 9, 940 947. https://doi.org/10.1038/nn1724.
Kim, Y.T., Seo, J.H., Song, H.J., Yoo, D.S., Lee, H.J., Lee, J., Lee, G., Kwon, E., Kim, J.G., Chang, Y., 2011. Neural correlates related to action observation in expert archers. Behav. Brain Res. 223, 342-347. https://doi.org/10.1016/j.bbr.2011.04.053.

Koch, M.A., Norris, D.G., Hund-Georgiadis, M., 2002. An investigation of functional and anatomical connectivity using magnetic resonance imaging. Neuroimage 16, 241250. https://doi.org/10.1006/nlmg.2001.1052

Kuruma, H., Watanabe, S., Ikeda, Y., Senoo, A., Kikuchi, Y., Abo, M., Yonemoto, K., 2007. Neural mechanism of self-initiated and externally triggered finger movements. J. Phys. Ther. Sci. 19, 103-109. https://doi.org/10.1589/jpts.19.103oi.org/10.

Landi, S.M., Baguear, F., Della-Maggiore, V., 2011. One weekofmotor adaptation induce structural changes in primary motor cortex that predict long-term memory one year later. J. Neurosci. 31, 11808-11813. https://doi.org/10.1523/jneurosci2253-11. 2011.

Latora,V., Marchiori,M., 2001.Efficientbehaviorofsmall-worldnetworks. Phys RevLett 87, 198701 1-198701 4. https://doi.org/10.1103/physrevlett87.198701.

Lerch, J.P., Yiu, A.P., Martinez-Canabal, A., Pekar, T., Bohbot, V.D., Frankland, P.W., Henkelman, R.M., Josselyn, S.A., Sled, J.G., 2011. Maze training in mice induces MRI-detectable brain shape changes specific to the type of learning. Neuroimage 54 , 2086-2095. https://doi.org/10.1016/j.neuroimage.2010.09.086.

Lewis, C.M., Baldassarre, A., Committeri, G., Romani, G.L., Corbetta, M., 2009. Learning sculpts the spontaneous activity of the resting human brain. PNAS 106, 17558 17563. https://doi.org/10.1073/pnas.0902455106.

Li, J., Luo, C., Peng, Y., Xie, Q., Gong, J., Dong, L., Lai, Y., Hi, H., Yao, D., 2014 Probability diffusion tractography reveals improvement of structural network in musician. PLoS One 8, e105508. https://doi.org/10.1371/journal.pone.0105508.

Li, G., He, H., Huang, M., Zhang, X., Lu, J., Lai, Y., Luo, C., Yao, D., 2015. Identifying enhanced cortico-basal ganglia loops associated with prolonged dance training. Sci. Rep. 5, 10271. https://doi.org/10.1038/srep10271.

Logan, G.D., 1988. Toward an instance theory of automatization. Psychol. Rev. 95, $492-527$.

Luo, C., Guo, Z.W., Lai, Y.X., Liao, W., Liu, Q., Kendrick, K.M., Yao, D.Z., Li, H., 2012 Musical training induces functional plasticity in perceptual and motor networks: insights from resting-state fMRI. PLoS One 7, e36568. https://doi.org/10.1371/ journal.pone.0036568.

Luo, C., Tu, S., Peng, Y., Gao, S., Li, J., Dong, L., Li, G., Lai, Y., Li, H., Yao, D., 2014. Longterm effects of musical training and functional plasticity in salience system. Neural Plasticity 180138, 1-13. https://doi.org/10.1155/2014/180138.

Ma,L., Narayana, S., Robin, D.A., Fox, P.T., Xiong, J., 2011. Changesoccurinrestingstate network of motor system during 4 weeks of motor skill learning. Neuroimage 58 , 226-233. https://doi.org/10.1016/j.neuroimage.2011.06.014.

Magill, R.A., 2010. Motor Learning and Control: Concepts and Applications. McGraw-Hill Higher Education, Columbus.

Makino, H., Hwang, E.U., Hedrick, N.H., Komiyama, T., 2016. Circuit mechanisms of sensorimotor learning. Neuron 92, 705-721. https://doi.org/10.1016/j.neuron.2016. 10.029 .

Manto, H., Bower, J.M., Conforto, A.B., Delgado-Garcia, J.M., da Guarda, S.N.F., Gerwig, M., Habas, C., Hagura, N., Ivry, R.B., Marien, P., Molinari, M., Naito, E., Nowak, D.A., Ben Taib, N.O., Pelisson, D., Tesche, C.D., Tilikete, C., Timmann, D., 2012. Consensus paper; roles of the cerebellum in motor-the diversity of ideas on cerebellum involvement in movement. Cerebellum 11, 457-487. https://doi.org/10.1007/s12311011-0331-9.

Mauk, M.D., Medina, J.F., Nores, W.L., Ohyama, T., 2000. Cerebellar function: coordination, learning or timing? Curr. Biol. 10, R522-R525. https://doi.org/10.1016/ S0960-9822(00), 00584-4.

McIntosh, A.R., Korostil, M., 2008. Interpretation of neuroimaging data basedon network concepts. Brain Imaging Behav. 2, 264-269. https://doi.org/10.1007/s11682-0089031-6.

McIntosh, A.R., Gonzalez-Lima, F., 1994. Structural equation modeling and its application to network analysis in functional brain imaging. Hum. Brain Mapp. 2, 2-22.

McKeown, M.J., Makeig, S., Brown, G.G., Jung, T.P., Kindermann, S.S., Bell, A.J., Sejnowski, T.J., 1998. Analysis of fMRI data by blind separation into independent spatial component. Hum. Brain Mapp. 6, 160-188.

Mechelli, A., Price, C.J., Friston, K.J., Ashburner, J., 2005. Voxel-based morphometry of the human brain: methods and applications. Curr. Med. Imaging Rev. 1, 105-113. https://doi.org/10.2174/1573405054038726.

Meister, I., Krings, T., Foltys, H., Boroojerdi, B., Muller, M., Topper, R., Thron, A., 2005 Effects of long-term practice and task complexity in musicians and nonmusicians performing simple and complex motor tasks: implications for cortical motor organization. Hum. Brain Mapp. 25, 345-352. https://doi.org/10.1002/hbm.20112.

Mennes, M., Kelly, C., Colcombe, S., Castellanos, F.X., Milham, M.P., 2013. The extrinsic and intrinsic functional architectures of the human brain are not equivalent. Cereb. Cortex 23, 223-229. https://doi.org/10.1093/cercor/bhs010.

Menon, V., Uddin, L.Q., 2010. Saliency, switching, attention and control: a network model of insula function. Brain Struct. Funct. 214, 655-667. https://doi.org/10. 1007/s00429-010-0262-0.

Miall, C., 2013. 10000 hours to perfection. Nat. Neurosci. 16, 1168-1169.

Nigmatullina, Y., Hellyer, P.J., Nachev, P., Sharp, D.J., Seemungal, B.M., 2015. The neuroanatomical correlates of training-related perceptuo-reflex uncoupling in dancers. Cereb. Cortex 25, 554-562. https://doi.org/10.1093/cercor/bht266.

O'Reilly, J.X., Beckmann, C.F., Tomassini, V., Ramnani, N., Johansen-Berg, H., 2010. Distinct and overlapping functional zones in the cerebellum defined by resting state functional connectivity. Cereb. Cortex 20, 953-965. https://doi.org/10.1093/cercor/ bhp157.

Paul, K.I., Cnossen, F., 2018. A cognitive neuroscience perspective on skill acquisition in cather-based interventions. In: Lanzer, P. (Ed.), Textbook of Catheter-based Cardiovascular Interventions. Springer, Cham, Switzerland, pp. 35-55. 
Pavlova, M.A., Sokolov, A.N., Bidet-Ildei, C., 2015. Sex differences in the neuromagnetic cortical response to biological motion. Cereb. Cortex 25, 3468-3474. https://doi.org/ 10.1093/cercor/bhu175.

Penhume, V.B., Steele, C.J., 2012. Parallel contributions of cerebellar, striatal and M1 mechanisms to motor sequence learning. Behav. Brain Res. 226, 579-591. https:// doi.org/10.1016/j.bbr.2011.09.044.

Petrini, K., Pollick, F.E., Dahl, S., McAleer,P., McKay, L.S., Rocchesso, D., Waadeland, C.H., Love, S., Avanzini, F., Puce, A., 2011. Actionexpertise reducesbrainactivity for audiovisual matching actions: an fMRI study with expert drummers. Neuroimage 56, 1480-1492. https://doi.org/10.1016/j.neuroimage.2011.03.009.

Picard, N., Matsuzaka, Y., Strick, P.L., 2013. Extended practice of a motor skill is associated with reduced metabolic activity in M1. Nat. Neurosci. 16, 1340-1347. https:// doi.org/10.1038/nn.3477.

Poldrack, R.A., 2015. Is "efficiency" a useful concept in cognitive neuroscience? Dev. Cogn. Neurosci. 11, 12-17. https://doi.org/10.1016/j.den.2014.06.001.

Poldrack, R.A., Desmond, J.E., Glover, G.H., Gabrieli, J.D., 1998. The neural basis of visual skill learning: an fMRI study of mirror reading. Cereb. Cortex 8, 1-10.

Reed, A., Riley, J., Carraway, R., Carrasco, A., Perez, C., Jakkamsetti, V., Kilgard, M.P., 2011. Cortical map plasticity improves learning but is not necessary for improved performance. Neuron 70, 121-131. https://doi.org/10.1016/j.neuron.2011.02.038.

Roebroeck, A., Formisano, E., Goebel, R., 2005. Mapping directed influence over the brain using Granger causality and fMRI. Neuroimage 25, 230-242. https://doi.org/ 10.1016/j.neuroimage.2004.11.017.

Seeley, W.W., Menon, V., Schatzberg, A.F., Keller, J., Glover, G.H., Kenna, H., Reiss, A.L., Greicius, M.D., 2007. Dissociable intrinsic connectivity networks for salience processing and executive control. J. Neurosci. 27, 2349-2356. https://doi.org/10.1523/ JNEUROSCI.5587-06.2007.

Shen, G., Zhang, J., Wang, H., Wu, Y., Zeng, Y., Du, X., 2014. Altered white matter architecture among college athletes: a diffusion tensor imaging study. J. East China Normal Univ. (Nat. Sci.) 4, 94-101.

Schmithorst, V.J., Wilke, M., 2002. Differences in white matter architecture between musicians and non-musicians: a diffusion tensor imaging study. Neurosci. Lett. 321, $57-60$.

Scholz, J., Klein, M.C., Behrens, T.E.J., Johansen-Berg, H., 2009. Training induces changes in white matter architecture. Nat. Neurosci. 12, 1370-1371. https://doi.org/ $10.1038 / \mathrm{nn} .2412$.

Skudlarski, P., Jagannathan, K., Calhoun, V.D., Hampson, M., Skudlarska, B.A., Pearlson, G., 2008. Measuring brain connectivity: diffusion tensor imaging validates resting state temporal correlations. Neuroimage 43, 554-561. https://doi.org/10.1016/j. neuroimage.2008.07.063

Smith, S.M., Fox, P.T., Miller, K.L., Glahn, D.C., Fox, P.M., Mackay, C.E., Filippini, N., Watkins, K.E., Toro, R., Laird, A.R., Beckmann, C.F., 2009. Correspondence of the brain's functional architecture during activation and rest. PNAS USA 106, 13040 13045. https://doi.org/10.1073/pnas.0905267106.

Sowell, E.R., Peterson, B.S., Kan, E., Woods, R.P., Yoshii, J., Bansal, R., Xu, D., Zhu, H., Thompson, P.M., Toga, A.W., 2007. Sex differences in cortical thickness mapped in 176 healthy individuals between 7 and 87 years of age. Cereb. Cortex 17, 1550-1560. https://doi.org/10.1093/cercor/bhl066.

Stam, C.J., van Straaten, E.C.W., 2012. The organization of physiological brain networks. Clin. Neurophysiol. 123, 1067-1087. https://doi.org/10.1016/j.clinph.2012.01.011.

Steele, C.J., Scholz, J., Douaud, G., Johansen-Berg, H., Penhune, V.B., 2012. Structural correlates of skilled performance on a motor sequence task. Front. Hum. Neurosci. 6, 289. https://doi.org/10.3389/fnhum.2012.00289.

Stephan, K.E., Harrison, L.M., Kiebel, S.J., David, O., Penny, W.D., Friston, K.J., 2007 Dynamic causal models of neural system dynamics: current state and future extensions. J. Biosci. 32, 129-144.

Stoodley, C.J., Schmahmann, J.D., 2009. Functional topography in the human cerebellum: a meta-analysis of neuroimaging studies. Neuroimage 44, 489-501. https:// doi.org/10.1016/j.neuroimage.2008.08.039.

Swisher, J.D., Gatenby, J.C., Gore, J.C., Wolfe, B.A., Moon, C.H., Kim, S.G., Tong, F, 2010. Multiscale pattern analysis of orientation-selective activity in the primary visual cortex. J. Neurosci. 30, 325-330. https://doi.org/10.1523/jneurosci.4811-09. 2010.

Takahashi, M., Hackney, D.B., Zhang, G., Wehrli, S.L., Wright, A.C., O'Brian, W.T., Uematsu, H., Wehrli, F.W., Selzer, M.E., 2002. Magnetic resonance microimaging of intraaxonal water diffusion in live excised lamprey spinal cord. PNAS USA 99 16192-16196. https://doi.org/10.1073/pnas.252249999.

Tan, X.Y., Pi, Y.L., Wang, J., Li, X.P., Zhang, L.L., Dai, W., Zhu, H., Ni, Z., Zhang, J., Wu, Y., 2017. Morphological and functional differences between athletes and novices in cortical neuronal networks. Front. Hum. Neurosci. 10, 660. https://doi.org/10.3389/ fnhum.2016.00660.

Taubert, M., Draganski, B., Anwander, A., Muller, K., Horstmann, A., Villringer, A., Ragert, P., 2010. Dynamic properties of human brain structure: learning-related changes in cortical areas and associated fiber connections. J. Neurosci. 30, 11670 11677. https://doi.org/10.1523/jneurosci.2567-10.2010.

Taubert, M., Lohmann, G., Margulies, D.S., Villringer, A., Ragert, P., 2011. Long-term effects of motor training on resting-state networks and underlying brain structure. Neuroimage 57, 1492-1498. https://doi.org/10.1016/j.neuroimage.2011.05.078.
Tomasi, D., Volkow, N.D., 2012. Gender differences in brain functional connectivity density. Hum. Brain Mapp. 33, 849-860. https://doi.org/10.1002/hbm.21252. Tuch, D.S., 2004. Q-ball imaging. Magn. Reson. Med. 52, 1358-1372.

Tuch, D.S., Reese, T.G., Wiegell, M.R., Makris, N., Belliveau, J.W., Wedeen, V.J., 2002. High angular resolution diffusion imaging reveals intravoxel white matter fiber heterogeneity. Magn. Reson. Med. 48, 577-582.

Tyc, F., Boyadjian, A., 2006. Cortical plasticity and motor activity studied with transcranial magnetic stimulation. Rev. Neurosci. 17, 469-496. https://doi.org/10.1515/ revneuro.2006.17.5.469.

Van Donkelaar, P., Stein, J.F., Passingham, R.E., Miall, R.C., 1999. Neuronal activity in the primate motor thalamus during visually triggered and internally generated limb movements. J. Neurophysiol. 82, 934-945.

Van Veel, V., Cohen, J.D., Botvinick, M.M., Stenger, V.A., Carter, C.S., 2001 . Anterior cingulate cortex, conflict monitoring, and levels of processing. Neuroimage 14 1302-1308. https://doi.org/10.1006/nimg.2001.0923.

Voelcker-Rehage, C., Niemann, C., 2013. Structural and functional brain changes related to different types of physical activity across the life span. Neurosci. Biobehav. Rev. 37, 2268-2295. https://doi.org/10.1016/j.neubiorev.2013.01.028.

Wang, Z., Dai, Z., Gong, G., Zhou, C., He, Y., 2014. Understanding structural-functional relationships in the human brain: a large-scale network perspective. Neuroscientist 21, 290-305. https://doi.org/10.1177/1073858414537560.

Wang, B., Fan, Y., Lu, M., Li, S., Song, Z., Peng, X., Zhang, R., Lin, Q., He, Y., Wang, J., Huang, R., 2013. Brain anatomical networks in world class gymnasts: a DTI tractography study. Neuroimage 65, 476-487. https://doi.org/10.1016/j.neuroimage. 2012.10.007.

Wang, B., Lu, M., Fan, Y., Wen, X., Zhang, R., Wang, B., Ma, Q., Song, Z., He, Y., Wang, J., Huang, R., 2016. Exploring brain functional plasticity in world class gymnasts: a network analysis. Brain Struct. Funct. 221, 3503-3519. https://doi.org/10.1007/ s00429-015-1116-6.

Wang, Z., Dai, Z., Gong, G., Zhou, C., He, Y., 2015. Understanding structural-functional relationships in the human brain: a large-scale network perspective. Neuroscientist 21, 290-305. https://doi.org/10.1177/1073858414537560.

Watts, D.J., Strogatz, S.H., 1998. Collective dynamics of 'small-world' networks. Nature 393, 440-442. https://doi.org/10.1038/30918.

Wedeen, V.J., Hagmann, P., Tseng, W.Y., Reese, T.G., Weisskoff, R.M., 2005. Mapping complex tissue architecture with diffusion spectrum magnetic resonance imaging. Magn. Reson. Med. 54, 1377-1386.

Wei, G., Luo, J., Li, Y., 2009. Brain structure in diving players on MR imaging studied with voxel-based morphometry. Prog. Nat. Sci. 19, 1397-1402. https://doi.org/10. 1016/j.pnsc.2008.12.009

Wei, G., Zhang, Y., Jiang, T., Luo, J., 2011. Increased cortical thickness in sports experts: a comparison of diving players with the controls. PLoS One 6, e17112. https://doi.org/ 10.1371/journal. pone.0017112.

Wenger, E., Brozzoli, C., Lindenberger, U., Lovden, M., 2017a. Expansion and renormalization of human brain structure during skill acquisition. Trends Cogn. Sci. 21, 930-939. https://doi.org/10.1016/j.tics.2017.09.008.

Wenger, E., Kuhn, S., Verrel, J., Martensson, J., Bodammer, N.C., Lindenberger, U., Lovden, M., 2017b. Repeated structural imaging reveals nonlinear progression of experience-dependent volume changes in human motor cortex. Cereb. Cortex 27, 2911-2925. https://doi.org/10.1093/cercor/bhw141.

Wiestler, T., Diedrichsen, J., 2013. Skill learning strengthens cortical representations of motor sequences. eLIFE 2, e00801. https://doi.org/10.7554/elife.00801.

Witelson, S.F., Glezer, I.I., Kigar, D.L., 1995. Women have greater density of neurons in posterior temporal cortex. J. Neurosci. 15, 3418-3428.

Woollett, K., Maguire, E.A., 2011. Acquiring "the knowledge" of London's layout drive structural brain changes. Curr. Biol. 21, 2109-2114. https://doi.org/10.1016/j.cub. 2011.11.018.

Xiong, J., Ma, L., Wang, B., Narayana, S., Duff, E.P., Egan, G.F., Fox, P.T., 2009. Longterm motor training induced changes in regional cerebral blood flow in both task and resting states. Neuroimage 45, 75-82. https://doi.org/10.1016/j.neuroimage.2008. 11.016.

Xu, T., Yu, X., Perlik, A.J., Tobin, W.F., Zweig, J.A., Tennant, K., Jones, T., Zuo, Y., 2009 Rapid formation and selective stabilisation of synapses for enduring motor memories. Nature 462, 915-919. https://doi.org/10.1038/nature08389.

Yang, J., 2015. The influence of motor expertise on the brain activity of motor task performance: a meta-analysis of functional magnetic resonance imaging studies. Cogn. Affect Behav. 15, 381-394. https://doi.org/10.3758/s13415-014-0329-0.

Zatorre, R.J., Chen, J.L., Penhune, V.B., 2007. When the brain plays music: auditorymotor interactions in music perception and production. Nat. Rev. Neurosci. 8, $547-$ 558. https://doi.org/10.1038/nrn2152.

Zatorre, R.J., Fields, R.D., Johansen-Berg, H., 2012. Plasticity in gray and white: neuroimaging changes in brain structure during learning. Nat. Neurosci. 15, 528-536. https://doi.org/10.1038/nn.3045.

Zhang, J., Dong, X., Wang, L., Zhao, L., Weng, Z., Zhang, T., Sui, J., Go, R., Huang, Q., Wu, J., Yan, T., 2018. Gender differences in global functional connectivity during facial emotion processing: a visual MMN study. Front. Behav. Neurosci. 12, 220. https://doi.org/10.3389/fnbeh.2018.00220. 